\title{
EFFICIENT SOLUTION OF OPTIMAL CONTROL PROBLEMS USING HYBRID SYSTEMS*
}

\author{
MIREILLE BROUCKE ${ }^{\dagger}$, MARIA DOMENICA DI BENEDETTO $\ddagger$, \\ STEFANO DI GENNARO§, AND ALBERTO SANGIOVANNI-VINCENTELLI
}

\begin{abstract}
We consider the synthesis of optimal controls for continuous feedback systems by recasting the problem to a hybrid optimal control problem: synthesize optimal enabling conditions for switching between locations in which the control is constant. An algorithmic solution is obtained by translating the hybrid automaton to a finite automaton using a bisimulation and formulating a dynamic programming problem with extra conditions to ensure non-Zenoness of trajectories. We show that the discrete value function converges to the viscosity solution of the Hamilton-JacobiBellman equation as a discretization parameter tends to zero.
\end{abstract}

Key words. optimal control, hybrid systems, bisimulation

AMS subject classifications. 49L20, 49L25, 65N99

DOI. $10.1137 /$ S0363012900383090

1. Introduction. The goal of this paper is the development of a computationally appealing technique for synthesizing optimal controls for continuous feedback systems $\dot{x}=f(x, u)$ by recasting the problem as a hybrid optimal control problem. The hybrid problem is obtained by approximating the control set $U \subset \mathbb{R}^{m}$ by a finite set $\Sigma \subset U$ and defining vector fields for the locations of the hybrid system of the form $f(x, \sigma)$, $\sigma \in \Sigma$; that is, the control is constant in each location. The hybrid control problem is to synthesize enabling conditions such that a target set $\Omega_{f} \subset \Omega$ is reached while a hybrid cost function is minimized, for each initial condition in a specified set $\Omega \subset \mathbb{R}^{n}$.

Casting the problem as a hybrid control problem is not necessarily a simplification because, while algorithmic approaches for solving the controller synthesis problem for specific classes of hybrid systems have appeared [33, 52], no general, efficient algorithm is available. To be able to solve the (nonlinear) hybrid optimal control problem, we must exploit some additional property. We have a feasible and appealing approach if we can translate the problem to an equivalent discrete problem, which abstracts completely the continuous behavior. This translation is possible if we can construct a finite bisimulation defined on the hybrid state space, that is, an equivalence relation that induces a partition in each hybrid automaton location that is consistent with the continuous dynamics of that location. A finite bisimulation can be constructed using the geometric approach reported in [10], based on the following key assumption: $n-1$ local (on $\Omega$ ) first integrals can be expressed analytically for each vector field $f(x, \sigma)$, $\sigma \in \Sigma$. This assumption is imposed in the transient phase of a feedback system's

* Received by the editors December 20, 2000; accepted for publication (in revised form) June 7 , 2004; published electronically April 14, 2005.

http://www.siam.org/journals/sicon/43-6/38309.html

$\dagger^{\dagger}$ Department of Electrical and Computer Engineering, University of Toronto, Toronto, ON, Canada M5S 3G4 (broucke@control.utoronto.ca).

¥Dip. di Ingegneria Elettrica, Università di L'Aquila, Poggio di Roio, 67040 L’Aquila, Italy, and Department of Electrical Engineering and Computer Sciences, University of California, Berkeley, CA 94720 (marika@eecs.berkeley.edu).

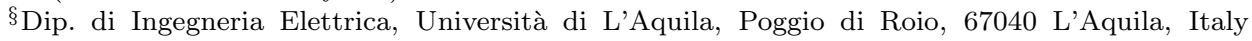
(digennar@dis.uniroma1.it).

"Department of Electrical Engineering and Computer Sciences, University of California, Berkeley, CA 94720 (alberto@eecs.berkeley.edu). 
response, when the vector field is nonvanishing and local first integrals always exist, although finding closed form expressions for them is not always easy or possible. Also, the assumption that the partition be a bisimulation is sufficient but not necessary for the overall approach.

If the assumption is met, then we can transform the hybrid system to a quotient system associated with the finite bisimulation, which is a finite automaton. The control problem posed on the finite automaton is to synthesize a discrete supervisor, providing a switching rule between automaton locations, that minimizes a discrete cost function approximating the original cost function, for each initial discrete state. We provide a dynamic programming solution to this problem, with extra constraints to ensure non-Zenoness of the closed-loop trajectories. By imposing non-Zeno conditions on the synthesis we obtain piecewise constant controls with a finite number of discontinuities in bounded time.

The discrete value function depends on the discretizations of $U$ and of $\Omega$ using the bisimulation. We quantify these discretizations by parameters $\delta$ and $\delta_{Q}$. The main theoretical contribution is to show that as $\delta, \delta_{Q} \rightarrow 0$, the discrete value function converges to the unique viscosity solution of the Hamilton-Jacobi-Bellman (HJB) equation.

There is a similarity between our approach to optimal control and regular synthesis, introduced in [8], in the sense that both restrict the class of controls to a set that has some desired property and both use a finite partition to define switching behavior. For linear systems, the results on regular synthesis are centered on the bang-bang principle [38], stating that a sufficient class of optimal controls is piecewise constant. If $U$ is a convex polyhedron, then the number of discontinuities of the control is bounded. There is no hope that general bang-bang results are available due to Fuller's example [21, 29]. Nevertheless, in many applications the optimal control is a piecewise continuous function, and methods of regular synthesis of such controls are worth investigating. Our paper focuses on piecewise constant controls and provides a constructive approach to obtaining a cell decomposition, in the spirit of regular synthesis, by using a finite bisimulation, which further allows us to formulate the synthesis problem on its quotient system - a finite automaton.

The idea of using a time abstract model formed by partitioning the continuous state space has been pursued in a number of papers recently. Stiver, Antsaklis, and Lemmon [48] and Yang, Lemmon, and Antsaklis [53] used a partition of the state space to convert a hybrid model to a discrete event system (DES). This enabled them to apply controller synthesis for DESs to synthesize a supervisor. While our approach is related to this methodology, it differs in that we provide conditions for obtaining the partition. In [41] hybrid systems consisting of a linear time-invariant system and a discrete controller that has access to a quantized version of the linear system's output is considered. The quantization results in a rectangular partition of the state space. This approach suffers from spurious solutions that must be trimmed from the automaton behavior. Hybrid optimal control problems have been studied in papers by Witsenhausen [51], Branicky, Borkar, and Mitter [9], and Bensoussan and Menaldi [6]. The first two studies concentrated on problems of well-posedness, necessary conditions, and existence of optimal solutions but did not provide algorithmic solutions. Bensoussan and Menaldi considered a more general model than ours that included continuous dynamics with a measurable control input and a discrete part with impulsive control. Control switches can be autonomous or controlled and may have time delays. They characterized the viscosity solution of a dynamic programming problem 
on their model. They constructed open-loop controls, whereas we obtain feedback controls, and they did not consider the numerical implementation.

There has recently been significant progress in developing numerical methods that incorporate geometric invariants of a dynamical or control system. In particular, in the area of geometric mechanics, numerical integrators have been developed that preserve the Hamiltonian, Lie group symmetries, and other integrals of motion [19, 27, $25,28,40]$. See [36] for an overview of problems where geometric structure is exploited in numerical methods. Our work represents the first general methodology in which geometric invariants are explicitly considered in the numerical solution of optimal control problems. The geometric structure that is present in the optimal control problem is encoded in the bisimulation partition. In effect, the two-step procedure of a time discretization followed by the state discretization via finite element methods that together lead to a fixed point formulation of the approximate solution of a continuous time optimal control problem is circumvented. Instead an exact representation of the time evolution of the system is encoded in the finite element partition, enabling a simplified and more efficient formulation.

The paper is organized as follows. In section 2 we state the optimal control problem, while in section 3 the associated hybrid system is given. In section 4 we review how the bisimulation is constructed. Section 5 formulates the proposed solution using bisimulation and dynamic programming. In section 6 we prove the main theoretical result. In section 7 we present an algorithmic solution of the dynamic programming problem including a formal justification of the algorithm's optimality. In section 8 we give two simple examples. Section 9 summarizes our findings.

2. Optimal control problem. Notation. First, we introduce notation. $\mathbf{1}(\cdot)$ is the indicator function. $\operatorname{cl}(A)$ denotes the closure of set $A .\|\cdot\|$ denotes the Euclidean norm. Let $C^{1}\left(\mathbb{R}^{n}\right)$ and $\mathcal{X}\left(\mathbb{R}^{n}\right)$ denote the sets of continuously differentiable realvalued functions and smooth vector fields on $\mathbb{R}^{n}$, respectively. $\phi_{t}\left(x_{0}, \mu\right)$ denotes the trajectory of $\dot{x}=f(x, \mu)$ starting from $x_{0}$ and using control $\mu(\cdot)$.

Let $U$ be a compact subset of $\mathbb{R}^{m} ; \Omega$ an open, bounded, connected subset of $\mathbb{R}^{n}$; and $\Omega_{f}$ a compact subset of $\Omega$. Define $\mathcal{U}_{m}$ to be the set of measurable functions mapping $\mathbb{R}^{+}$to $U$. We define the minimum hitting time $T: \mathbb{R}^{n} \times \mathcal{U}_{m} \rightarrow \mathbb{R}^{+}$by

$$
T(x, \mu):= \begin{cases}\infty & \text { if }\left\{t \mid \phi_{t}(x, \mu) \in \Omega_{f}\right\}=\emptyset, \\ \min \left\{t \mid \phi_{t}(x, \mu) \in \Omega_{f}\right\} & \text { otherwise. }\end{cases}
$$

A control $\mu \in \mathcal{U}_{m}$ specified on $[0, T]$ is admissible for $x \in \Omega$ if $\phi_{t}(x, \mu) \in \Omega$ for all $t \in[0, T]$. The set of admissible controls for $x$ is denoted $\mathcal{U}_{x}$. Let

$$
\mathcal{R}:=\left\{x \in \Omega \mid \exists \mu \in \mathcal{U}_{x} . T(x, \mu)<\infty\right\} .
$$

We consider the following stationary optimal control problem. Given $y \in \Omega$,

$$
\begin{array}{rlr}
\text { minimize } & J(y, \mu)=\int_{0}^{T(y, \mu)} L(x(t), \mu(t)) d t+h(x(T(y, \mu))) \\
\text { subject to } & \dot{x}=f(x, \mu), \quad \text { a.e. } t \in[0, T(y, \mu)], \\
& x(0)=y
\end{array}
$$

among all admissible controls $\mu \in \mathcal{U}_{y} . J: \mathbb{R}^{n} \times \mathcal{U}_{m} \rightarrow \mathbb{R}$ is the cost-to-go function, $h: \mathbb{R}^{n} \rightarrow \mathbb{R}$ is the terminal cost, and $L: \mathbb{R}^{n} \times \mathbb{R}^{m} \rightarrow \mathbb{R}$ is the instantaneous cost. At $T(y, \mu)$ the terminal cost $h(x(T(y, \mu)))$ is incurred and the dynamics are stopped. The control objective is to reach $\Omega_{f}$ from $y \in \Omega$ with minimum cost. 
Assumption 2.1 .

(1) $f: \mathbb{R}^{n} \times \mathbb{R}^{m} \rightarrow \mathbb{R}^{n}$ satisfies $\left\|f\left(x^{\prime}, u^{\prime}\right)-f(x, u)\right\| \leq L_{f}\left[\left\|x^{\prime}-x\right\|+\left\|u^{\prime}-u\right\|\right]$ for some $L_{f}>0$. Let $M_{f}$ be the upper bound of $\|f(x, u)\|$ on $\Omega \times U$.

(2) $L: \mathbb{R}^{n} \times \mathbb{R}^{m} \rightarrow \mathbb{R}$ satisfies $\left|L\left(x^{\prime}, u^{\prime}\right)-L(x, u)\right| \leq L_{L}\left[\left\|x^{\prime}-x\right\|+\left\|u^{\prime}-u\right\|\right]$ and $1 \leq L(x, u) \leq M_{L}, x \in \Omega, u \in U$, for some $L_{L}, M_{L}>0$.

(3) $h: \mathbb{R}^{n} \rightarrow \mathbb{R}$ satisfies $\left|h\left(x^{\prime}\right)-h(x)\right| \leq L_{h}\left\|x^{\prime}-x\right\|$ for some $L_{h}>0$, and $h(x) \geq 0$ for all $x \in \Omega$. Let $M_{h}$ be the upper bound of $|h(x)|$ on $\Omega$.

Remark 2.1. These assumptions ensure existence of solutions to (2.3) and uniqueness of the trajectories $\phi_{t}(x, \mu)$. Weaker assumptions are possible; see [4].

The value function or optimal cost-to-go function $V: \mathbb{R}^{n} \rightarrow \mathbb{R}$ is given by

$$
V(y)=\inf _{\mu \in \mathcal{U}_{y}} J(y, \mu)
$$

for $y \in \Omega \backslash \Omega_{f}$ and by $V(y)=h(y)$ for $y \in \Omega_{f}$. A control $\mu$ is called $\epsilon$-optimal for $x$ if $J(x, \mu) \leq V(x)+\epsilon$.

It is well known [20] that $V$ satisfies the HJB equation

$$
-\inf _{u \in U}\left\{L(x, u)+\frac{\partial V}{\partial x} f(x, u)\right\}=0
$$

at each point of $\mathcal{R}$ at which it is differentiable. The HJB equation is an infinitesimal version of the equivalent dynamic programming principle (DPP), which says that

$$
\begin{aligned}
& V(x)=\inf _{\mu \in \mathcal{U}_{x}}\left\{\int_{0}^{t} L\left(\phi_{s}(x, \mu), \mu(s)\right) d s+V\left(\phi_{t}(x, \mu)\right)\right\}, \quad x \in \Omega \backslash \Omega_{f}, \\
& V(x)=h(x), \quad x \in \Omega_{f} .
\end{aligned}
$$

The subject of assiduous effort has been that the HJB equation may not have a $C^{1}$ solution. This gap in the theory was closed by the introduction of the concept of viscosity solution $[32,14]$, which can be shown to provide the unique solution of (2.5) without any differentiability assumption. In particular, a bounded uniformly continuous function $V$ is called a viscosity solution of HJB provided, for each $\psi \in$ $C^{1}\left(\mathbb{R}^{n}\right)$, the following hold:

(i) if $V-\psi$ attains a local maximum at $x_{0} \in \mathbb{R}^{n}$, then

$$
-\inf _{u \in U}\left\{L\left(x_{0}, u\right)+\frac{\partial \psi}{\partial x}\left(x_{0}\right) f\left(x_{0}, u\right)\right\} \leq 0
$$

(ii) if $V-\psi$ attains a local minimum at $x_{1} \in \mathbb{R}^{n}$, then

$$
-\inf _{u \in U}\left\{L\left(x_{1}, u\right)+\frac{\partial \psi}{\partial x}\left(x_{1}\right) f\left(x_{1}, u\right)\right\} \geq 0 .
$$

Assumption 2.2. For every $\epsilon>0$ and $x \in \mathcal{R}$, there exists $N_{\epsilon}>0$ and an admissible piecewise constant $\epsilon$-optimal control $\mu$ having at most $N_{\epsilon}$ discontinuities and such that $\phi_{t}(x, \mu)$ is transverse to $\partial \Omega_{f}$.

The transversality assumption implies that the viscosity solution is continuous at the boundary of the target set, a result needed in proving uniform continuity of $V$ 
over a finite horizon. The assumption can be replaced by a small-time controllability condition. For a treatment of small-time controllability and compatibility of the terminal cost with respect to continuity of the value function, see [4]. The finite switching assumption holds under mild assumptions, such as Lipschitz continuity of the vector field and cost functions, and is based on approximating measurable functions by piecewise constant functions.

3. Hybrid system. The approach we propose for solving the continuous optimal control problem first requires a mapping to a hybrid system and, second, employs a bisimulation of the hybrid system to formulate a dynamic programming problem on the quotient system. In this section we define the hybrid system. First, we discretize $U$ by defining a finite set $\Sigma_{\delta} \subset U$ which has a mesh size

$$
\delta:=\sup _{u \in U} \min _{\sigma \in \Sigma_{\delta}}\|u-\sigma\| .
$$

We define the hybrid automaton $H:=\left(\Sigma \times \mathbb{R}^{n}, \Sigma_{\delta}, D, E_{h}, G\right)$ with the following components.

State set. $\Sigma \times \mathbb{R}^{n}$ consists of the finite set $\Sigma=\Sigma_{\delta} \cup\left\{\sigma_{f}\right\}$ of control locations and $n$ continuous variables $x \in \mathbb{R}^{n}$. $\sigma_{f}$ is a terminal location when the optimal control problem is stopped and the target set is reached. The controller for $\sigma_{f}$ may, for instance, be a linear feedback designed using the linearization of the system.

Events. $\Sigma_{\delta}$ is a finite set of control events.

Vector fields. $D: \Sigma \rightarrow \mathcal{X}\left(\mathbb{R}^{n}\right)$ is a function assigning an autonomous vector field to each location. We use the notation $D(\sigma)=f_{\sigma}$.

Control switches. $E_{h} \subset \Sigma \times \Sigma$ is a set of control switches. $e=\left(\sigma, \sigma^{\prime}\right)$ is a directed edge between a source location $\sigma$ and a target location $\sigma^{\prime}$. If $E_{h}(\sigma)$ denotes the set of edges that can be enabled at $\sigma \in \Sigma$, then $E_{h}(\sigma):=\left\{\left(\sigma, \sigma^{\prime}\right) \mid \sigma^{\prime} \in \Sigma \backslash \sigma\right\}$ for $\sigma \in \Sigma_{\delta}$ and $E_{h}\left(\sigma_{f}\right)=\emptyset$. Thus, from a source location not equal to $\sigma_{f}$, there is an edge to every other location (but not itself), while location $\sigma_{f}$ has no outgoing edges.

Enabling conditions. $G: E_{h} \rightarrow\left\{g_{e}\right\}_{e \in E_{h}}$ is a function assigning to each edge an enabling (or guard) condition $g \subset \mathbb{R}^{n}$. We use the notation $G(e)=g_{e}$. The optimal enabling conditions are unknown and must be synthesized.

3.1. Semantics. A state is a pair $(\sigma, x), \sigma \in \Sigma$ and $x \in \mathbb{R}^{n}$. In location $\sigma \in \Sigma_{\delta}$ the continuous state evolves according to the vector field $f(x, \sigma)$. In location $\sigma_{f}$, the vector field is $\dot{x}=f\left(x, \mu_{f}\right)$, where $\mu_{f}$ is the (not necessarily constant) control of the terminal location. Trajectories of $H$ evolve in steps of two types. A $\sigma$-step is a binary relation $\stackrel{\sigma}{\rightarrow} \subset\left(\Sigma \times \mathbb{R}^{n}\right) \times\left(\Sigma \times \mathbb{R}^{n}\right)$, and we write $(\sigma, x) \stackrel{\sigma^{\prime}}{\rightarrow}\left(\sigma^{\prime}, x^{\prime}\right)$ iff $(1) e=\left(\sigma, \sigma^{\prime}\right) \in E_{h}$, (2) $x \in g_{e}$, and (3) $x=x^{\prime}$. A $t$-step is a binary relation $\stackrel{t}{\rightarrow} \subset\left(\Sigma \times \mathbb{R}^{n}\right) \times\left(\Sigma \times \mathbb{R}^{n}\right)$, and we write $(\sigma, x) \stackrel{t}{\rightarrow}\left(\sigma^{\prime}, x^{\prime}\right)$ iff $(1) \sigma=\sigma^{\prime}$ and (2) for some $t \geq 0, x^{\prime}=\phi_{t}(x, \sigma)$, where $\dot{\phi}_{t}(x)=f\left(\phi_{t}(x, \sigma), \sigma\right)$. Enabling conditions are forced in that an edge is taken instantaneously and as soon as it is enabled.

Example 3.1. Consider the time optimal control problem for the system

$$
\begin{aligned}
& \dot{x}_{1}=x_{2}, \\
& \dot{x}_{2}=u .
\end{aligned}
$$

Suppose $\Omega=(-1,1) \times(-1,1)$ and $\Omega_{f}=\bar{B}_{\epsilon}(0)$, the closed epsilon ball centered at 0 . The cost-to-go function is $J(x, \mu)=\int_{0}^{T(x, \mu)} d t$ and $U=\{u:|u| \leq 1\}$. We select 


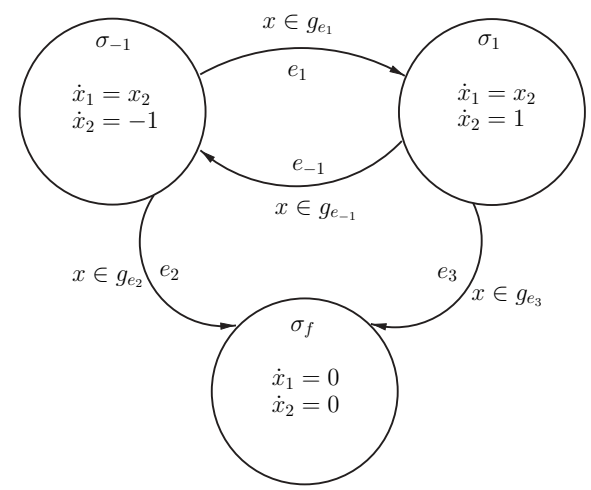

FIG. 3.1. Hybrid automaton for time optimal control of a double integrator system.

$\Sigma_{\delta}=\{-1,1\}$, so that $\delta=1$. The hybrid system is show in Figure 3.1. The state set is $\left\{\sigma_{-1}=-1, \sigma_{1}=1, \sigma_{f}\right\} \times \mathbb{R}^{2} . g_{e_{-1}}$ and $g_{e_{1}}$ are unknown and must be synthesized, while $g_{e_{2}}=g_{e_{3}}=\Omega_{f}$.

4. Bisimulation. Let $\lambda$ represent an arbitrary time interval. A bisimulation of $H$ is an equivalence relation $\simeq \subset\left(\Sigma_{\delta} \times \mathbb{R}^{n}\right) \times\left(\Sigma_{\delta} \times \mathbb{R}^{n}\right)$ such that for all states $p_{1}, p_{2} \in \Sigma_{\delta} \times \mathbb{R}^{n}$, if $p_{1} \simeq p_{2}$ and $\beta \in \Sigma_{\delta} \cup\{\lambda\}$, then if $p_{1} \stackrel{\beta}{\rightarrow} p_{1}^{\prime}$, there exists $p_{2}^{\prime}$ such that $p_{2} \stackrel{\beta}{\rightarrow} p_{2}^{\prime}$ and $p_{1}^{\prime} \simeq p_{2}^{\prime}$. See Figure 4.1. Intuitively, a bisimulation is an equivalence relation defining a partition on the hybrid state space that preserves reachability over $\sigma$-steps and time steps. However, the definition leaves ambiguity about how the partition should be obtained. Alur and Dill [1] gave a construction for timed automata that was based on the first integrals of the continuous dynamics and on the syntax of the enabling and reset conditions. Their approach was first generalized in [10]. Time evolution of the original system is modeled as untimed transitions from equivalence class to equivalence class in the quotient system associated with the bisimulation. Transitions between locations of the hybrid automaton appear also as transitions in the quotient system. Thus if there is a finite number of equivalence classes of $\simeq$, then a finite transition system or finite automaton is obtained which gives a time abstract model of the original system, with reachability properties exactly preserved. For a more thorough discussion of results on bisimulations for hybrid systems, see $[26,2]$.

We declare the set of "interesting" equivalence classes of $\simeq$, which is assumed to be finite and is denoted $Q$, to be those that intersect $\Sigma_{\delta} \times \operatorname{cl}(\Omega)$. For each $q \in Q$ we define a distinguished point $(\sigma, \xi) \in q$, and we use the notation $q=[(\sigma, \xi)]$.

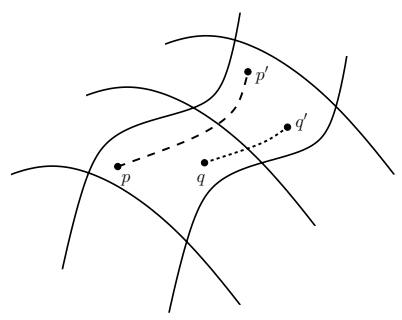

FIG. 4.1. Illustration of the definition of bisimulation. 
We define a mesh size on $Q$ by

$$
\delta_{Q}=\max _{q \in Q} \sup _{(\sigma, x),(\sigma, y) \in q}\|x-y\| .
$$

For each $q=[(\sigma, \xi)] \in Q$ we associate the duration $\tau_{q}$, the maximum time to traverse $q$ using constant control $\sigma$. That is,

$$
\tau_{q}=\sup _{(\sigma, x),(\sigma, y) \in q}\left\{t \mid y=\phi_{t}(x, \sigma)\right\} .
$$

This extra data associated with the bisimulation is required in our problem to obtain approximations of the cost functions $L$ and $h$.

4.1. Geometric construction. We review our method for obtaining finite bisimulations [10] which relies on the following assumptions on the vector fields on $\Omega$.

Assumption 4.1.

(1) For each $\sigma \in \Sigma_{\delta}$, there exist $n-1 C^{1}$ functions $\gamma_{i}^{\sigma}: \Omega \rightarrow \mathbb{R}, i=1, \ldots, n-1$, whose time derivative along solutions of $\dot{x}=f(x, \sigma)$ in $\Omega$ is zero.

(2) There exists $m_{f}>0$ such that $\|f(x, u)\| \geq m_{f}$ for all $x \in \operatorname{cl}(\Omega), u \in U$.

Remark 4.1. There is an uncontested view promulgated by Poincaré that differential equations possessing a complete set of first integrals, i.e., completely integrable systems, are the exception rather than the norm. This has led to some confusion about when one can or cannot find first integrals for non-Hamiltonian systems. The primary source of confusion comes from the multiple meanings of the term integrability. It appears as a Liouville integrability (the version alluded to by Poincaré and further developed by Arnold [3]), local integrability, algebraic integrability, etc. A type of integrability suitable for non-Hamiltonian systems was proposed by Chavarriga et al. [13] with the terminology weak integrability (to contrast with strong integrability in the sense of Liouville). Weak integrability is meant to capture that many systems do not exhibit complex behavior such as chaos, even if they are not Hamiltonian.

Let $\dot{x}=f(x)$ be a differential equation with domain of definition $\mathcal{D} \subset \mathbb{R}^{n}$, and let $\mathcal{O}$ be a set of orbits of the system such that $\mathcal{D} \backslash \mathcal{O}$ is open. Following [13], we say a $C^{1}$ function $\gamma: \mathcal{D} \backslash \mathcal{O} \rightarrow \mathbb{R}$ is a weak first integral of the system $\dot{x}=f(x)$ if $\gamma$ is constant on each solution of the system contained in $\mathcal{D} \backslash \mathcal{O}$ and $\gamma$ is nonconstant on any open subset of $\mathcal{D} \backslash \mathcal{O}$. A system is said to be weakly integrable if it has $n-1$ functionally independent (on $\mathcal{D} \backslash \mathcal{O}$ ) weak first integrals.

The relaxation of the requirement that the first integral be a differentiable function on the entire domain of the differential equation means that, for instance, all linear systems are weakly integrable [11], whereas only the Hamiltonian linear systems (centers and saddles in the case of second order linear systems) are integrable in the strong sense. Assumption 4.1(1) is a weak integrability assumption.

There are many methods for finding first integrals, including Lie group symmetry analysis [7, 37], Lax pairs, Painleve analysis, and the Frobenius theorem, among others $[16,22,46,49]$. A general reference and overview of the methods can be found in [24]. The best-known result for symbolic computation of first integrals is the Prelle-Singer procedure [39]. Reduce and Macsyma implementations of the Prelle-Singer procedure are described in $[34,46]$, while an implementation in higher dimensions is described in [35]. Algorithms for finding polynomial first integrals are described in [43, 44].

A bisimulation of $\Sigma_{\delta} \times \mathbb{R}^{n}$ is found by first constructing partitions for each location of $H$ such that reachability properties are preserved over time steps. In section 5 we describe how to accomodate $\sigma$-steps in the quotient system. To obtain a partition 

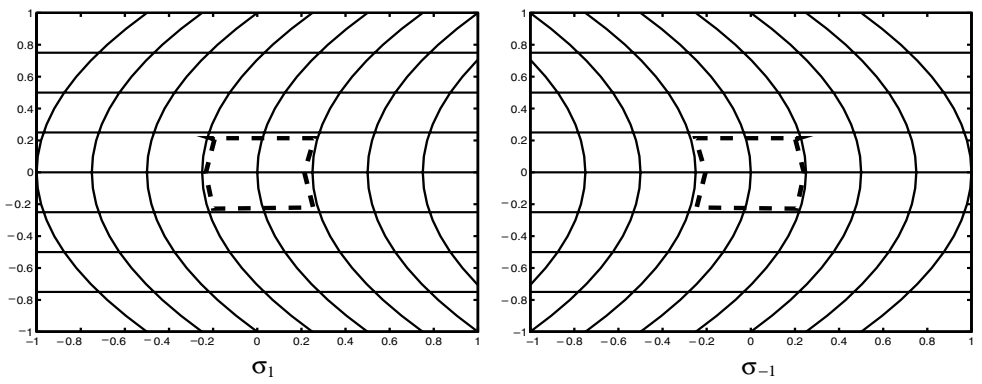

FIG. 4.2. Partitions for states $\sigma_{1}$ and $\sigma_{-1}$ of the hybrid automaton of Figure 3.1.

consistent with the dynamics of location $\sigma \in \Sigma_{\delta}$ we use the level sets of the $n-1$ first integrals $\gamma_{i}^{\sigma}(x)=y_{i}^{\sigma}, i=1, \ldots, n-1$, to bound the flow in $n-1$ independent directions, thus obtaining tubes of trajectories with a rectangular cross section. Next, the level sets of a submersion $\gamma_{n}^{\sigma}=y_{n}^{\sigma}$ that is transverse to the flow of $\dot{x}=f(x, \sigma)$ are used to divide the tube of trajectories into boxes, so that $\left(y_{1}^{\sigma}, \ldots, y_{n}^{\sigma}\right)$ form a set of Euclidean coordinates $\gamma^{\sigma}: \Omega \rightarrow[-1,1]^{n}$ on $\Omega$. That is, we assume that the level sets of $\gamma_{i}^{\sigma}$ foliate the set $\Omega$ (see [30] for background on foliations) and, by appropriate scaling, their level values lie between -1 and 1 on $\Omega$. We discretize the foliations associated with each $\gamma_{i}^{\sigma}$ by selecting a finite number of level values. More precisely, fix $k \in Z^{+}$and let $\Delta=\frac{1}{2^{k}}$. Define

$$
C_{k}=\{0, \pm \Delta, \pm 2 \Delta, \ldots, \pm 1\} .
$$

Each $y_{i}^{\sigma}=c$ for $c \in C_{k}, i=1, \ldots, n$, defines a hyperplane in $\mathbb{R}^{n}$ denoted $\tilde{W}_{i, c}^{\sigma}$ and a submanifold $W_{i, c}^{\sigma}=\left(\gamma^{\sigma}\right)^{-1}\left(\tilde{W}_{i, c}^{\sigma}\right)$. The collection of submanifolds for $\sigma \in \Sigma_{\delta}$ is

$$
\mathcal{W}_{k}^{\sigma}=\left\{W_{i, c}^{\sigma} \mid c \in C_{k}, i \in\{1, \ldots, n\}\right\} .
$$

$\Omega \backslash \mathcal{W}_{k}^{\sigma}$ is the union of $2^{n(k+1)}$ disjoint open sets $\mathcal{V}_{k}^{\sigma}=\left\{V_{j}^{\sigma}\right\}$. We define an equivalence relation $\simeq^{e}$ on $\mathbb{R}^{n}$ as follows. $y \simeq^{e} y^{\prime}$ iff

(1) $y \notin[-1,1]^{n}$ iff $y^{\prime} \notin[-1,1]^{n}$, and

(2) if $y, y^{\prime} \in[-1,1]^{n}$, then for each $i=1, \ldots, n, y_{i} \in(c, c+\Delta)$ iff $y_{i}^{\prime} \in(c, c+\Delta)$, and $y_{i}=c$ iff $y_{i}^{\prime}=c$ for all $c \in C_{k}$.

We define the equivalence relation $\simeq$ on $\Sigma_{\delta} \times \mathbb{R}^{n}$ as follows. $(\sigma, x) \simeq\left(\sigma^{\prime}, x^{\prime}\right)$ iff (1) $\sigma=\sigma^{\prime}$ and (2) $\gamma^{\sigma}(x) \simeq^{e} \gamma^{\sigma}\left(x^{\prime}\right)$.

Remark 4.2. A consequence of this construction is that if any trajectory of $H$ passing through $q \in Q$ spends zero time in it, then $\tau_{q}=0$.

Example 4.1. Continuing Example 3.1, a first integral for vector field $\dot{x}_{1}=x_{2}$, $\dot{x}_{2}=1$ is $x_{1}-\frac{1}{2} x_{2}^{2}=c_{1}, c_{1} \in \mathbb{R}$. For $\dot{x}_{1}=x_{2}, \dot{x}_{2}=-1$ a first integral is $x_{1}+\frac{1}{2} x_{2}^{2}=c_{2}$, $c_{2} \in \mathbb{R}$. We select a transverse foliation for each vector field, given by $x_{2}=c_{3}$. Partitions for locations $\sigma_{1}$ and $\sigma_{-1}$ and $\Omega=(-1,1) \times(-1,1)$ are shown in Figure 4.2. The equivalence classes of $\simeq$ are pairs consisting of a control event in $\Sigma_{\delta}$ and of the interiors of regions, open line segments and curves forming the boundaries of two regions, and the points at the corners of regions. $\tau=0$ for the segments transverse to the flow and the corner points. $\tau=\Delta$ for the interiors of regions and segments tangential to the flow, where $\Delta=.25$ in Figure 4.2.

5. Discrete problem. In this section we transform the hybrid optimal synthesis problem to a dynamic programming problem on a nondeterministic finite automaton. 


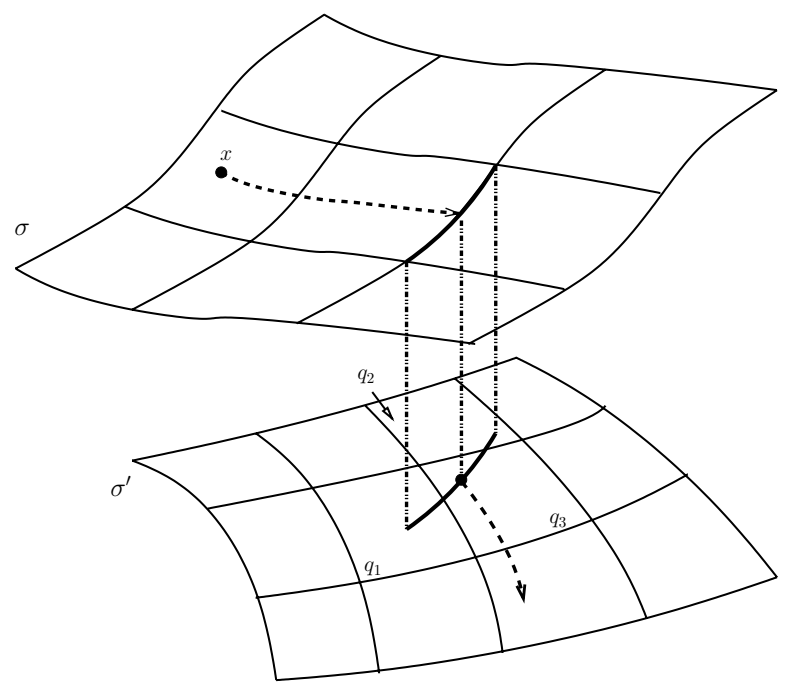

FIG. 5.1. Partitions for states $\sigma$ and $\sigma^{\prime}$ of a hybrid automaton, and the resulting nondeterminism in $A$.

Consider the class of nondeterministic finite automata with cost structure represented by the tuple

$$
A=\left(Q, \Sigma_{\delta}, E, \hat{L}, \hat{h}\right) .
$$

$Q$ is the finite state set, as defined above, and $\Sigma_{\delta}$ is the set of control events as before. $E \subseteq Q \times Q$ is the transition relation encoding $t$-steps and $\sigma$-steps of $H .\left(q, q^{\prime}\right) \in E$, where $q=[(\sigma, \xi)]$ and $q^{\prime}=\left[\left(\sigma^{\prime}, \xi^{\prime}\right)\right]$ if either (a) $\sigma=\sigma^{\prime}$, there exists $x \in \Omega$ such that $(\sigma, x) \in q$, and there exists $\tau>0$ such that for all $t \in[0, \tau],\left(\sigma, \phi_{t}(x, \sigma)\right) \in q$ and $\left(\sigma, \phi_{\tau+\epsilon}(x, \sigma)\right) \in q^{\prime}$ for arbitrarily small $\epsilon>0$; (b) $\sigma=\sigma^{\prime}$, there exists $x \in \Omega$ such that $(\sigma, x) \in q$, and there exists $\tau>0$ such that for all $t \in[0, \tau),\left(\sigma, \phi_{t}(x, \sigma)\right) \in q$ and $\left(\sigma, \phi_{\tau}(x, \sigma)\right) \in q^{\prime}$; or (c) $\sigma \neq \sigma^{\prime}$ and there exists $x \in \Omega$ such that $(\sigma, x) \in q$ and $\left(\sigma^{\prime}, x\right) \in q^{\prime}$. Cases (a) and (b) say that from a point in $q, q^{\prime}$ is the first state (different from $q$ ) reached after following the flow of $f(x, \sigma)$ for some time. Case (c) says that an edge exists between $q$ and $q^{\prime}$ if their projections to $\mathbb{R}^{n}$ have nonempty intersection.

Remark 5.1. The requirement that there be an edge from $q$ to $q^{\prime}$ if their projections to $\mathbb{R}^{n}$ have nonempty intersection is illustrated in Figure 5.1. We have partitions for controls $\sigma$ and $\sigma^{\prime}$, respectively. In the partition for $\sigma$, suppose a trajectory starting at $x$ flows in time using control $\sigma$ until state $q$ of $A$ is reached, at which time the control is set to $\sigma^{\prime}$. The possible states of $A$ that can be reached from $q$ are $q_{1}, q_{2}, q_{3}$, and the one-dimensional equivalence classes between them. Hence, edges corresponding to these possible futures for the trajectory must be included in the definition of $A$. A consequence is that multiple trajectories of $A$ can be defined starting from an initial state. One can think of this construction as overapproximating the identity map in terms of the equivalence classes of $\simeq$. This is the source of nondeterminacy of $A$.

Let $e=\left(q, q^{\prime}\right)$ with $q=[(\sigma, \xi)]$ and $q^{\prime}=\left[\left(\sigma^{\prime}, \xi^{\prime}\right)\right] . \hat{L}: E \rightarrow \mathbb{R}$ is the discrete instantaneous cost given by

$$
\hat{L}(e):= \begin{cases}\tau_{q} L(\xi, \sigma) & \text { if } \sigma=\sigma^{\prime} \\ 0 & \text { if } \sigma \neq \sigma^{\prime}\end{cases}
$$


$\hat{h}: Q \rightarrow \mathbb{R}$ is the discrete terminal cost given by

$$
\hat{h}(q):=h(\xi) .
$$

The domain of $\hat{h}$ can be extended to $\Omega$, with a slight abuse of notation, by

$$
\hat{h}(x):=\hat{h}(q),
$$

where $q=\arg \min _{q^{\prime}}\left\{\left\|x-\xi^{\prime}\right\| \mid q^{\prime}=\left[\left(\sigma^{\prime}, \xi^{\prime}\right)\right]\right\}$. Finally, $Q_{f}$ is the target set given by the overapproximation of $\Omega_{f}$,

$$
Q_{f}=\left\{q \in Q \mid \exists x \in \Omega_{f} \text { s.t. }(\sigma, x) \in q\right\} .
$$

5.1. Semantics. A transition or step of $A$ from $q=[(\sigma, \xi)] \in Q$ to $q^{\prime}=$ $\left[\left(\sigma^{\prime}, \xi^{\prime}\right)\right] \in Q$ is denoted $q \stackrel{\sigma^{\prime}}{\rightarrow} q^{\prime}$. If $\sigma \neq \sigma^{\prime}$, the transition is referred to as a control switch; otherwise, it is referred to as a time step. If $E(q)$ is the set of edges that can be enabled from $q \in Q$, then for $\sigma \in \Sigma_{\delta}$,

$$
E_{\sigma}(q)=\left\{e \in E(q) \mid e=\left(q, q^{\prime}\right), q=[(\sigma, \xi)], q^{\prime}=\left[\left(\sigma^{\prime}, \xi^{\prime}\right)\right]\right\} .
$$

If $\left|E_{\sigma}(q)\right|>1$, then we say that $e \in E_{\sigma}(q)$ is unobservable in the sense that when control event $\sigma$ is issued, it is unknown which edge among $E_{\sigma}(q)$ is taken. If $\sigma=\sigma^{\prime}$, then $\left|E_{\sigma}(q)\right|=1$, by the uniqueness of solutions of ODEs and by the definition of bisimulation.

A control policy $c: Q \rightarrow \Sigma_{\delta}$ is a map assigning a control event to each state; $c(q)=\sigma$ is the control event issued when the state is at $q$. A trajectory $\pi$ of $A$ over $c$ is a sequence $\pi=q_{0} \stackrel{\sigma_{1}}{\rightarrow} q_{1} \stackrel{\sigma_{2}}{\rightarrow} q_{2} \stackrel{\sigma_{3}}{\rightarrow} \ldots, q_{i} \in Q$. A trajectory is non-Zeno if between any two nonzero duration time steps there is a finite number of control switches. Note that this definition is slightly different from the traditional definition of non-Zeno trajectories of $H$ [26], in which it is assumed that time steps always have a nonzero duration. Here zero-duration time steps can occur. Let $\Pi_{c}(q)$ be the set of trajectories starting at $q$ and applying control policy $c$, and let $\tilde{\Pi}_{c}(q)$ be the set of trajectories starting at $q$, applying control policy $c$, and eventually reaching $Q_{f}$. If for every $q \in Q, \pi \in \Pi_{c}(q)$ is non-Zeno, then we say $c$ is an admissible control policy. The set of all admissible control policies for $A$ is denoted $\mathcal{C}$.

A control policy $c$ is said to have a loop if $A$ has a trajectory $q_{0} \stackrel{c\left(q_{0}\right)}{\longrightarrow} q_{1} \stackrel{c\left(q_{1}\right)}{\rightarrow}$ $\ldots \stackrel{c\left(q_{m-1}\right)}{\rightarrow} q_{m}=q_{0}, q_{i} \in Q$. A control policy has a Zeno loop if it has a loop made up of control switches and/or zero duration time steps (i.e., $\tau_{q}=0$ ) only.

Lemma 5.1. A control policy $c$ is admissible iff it has no Zeno loops.

Proof. First we show that a nondeterministic automaton with non-Zeno trajectories has a control policy without Zeno loops. Suppose not. Then a trajectory starting on a state belonging to the loop can take infinitely many steps around the loop before taking a nonzero duration time step. Such a trajectory must necessarily include a control switch (since a zero duration time step is always followed either by a nonzero duration time step or a control switch). Since this control switch occurs infinitely often in a finite time interval, the trajectory is Zeno, a contradiction.

Second, we show that a control policy without Zeno loops implies non-Zeno trajectories. Suppose not. Consider a Zeno trajectory that takes an infinite number of control switches in some finite time interval. Because there are a finite number of states in $Q$, by the Dirichlet principle [31], one of the states must be repeated in the sequence of states visited during the infinite number of control switches. Note that 


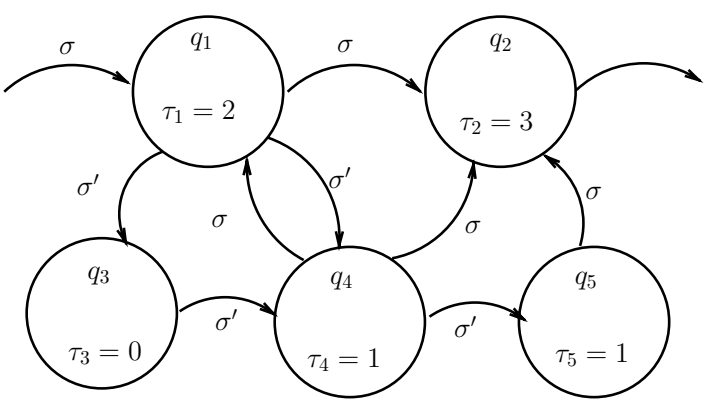

FIG. 5.2. Fragment of automaton with a zero duration time step.

this sequence can include zero duration time steps. This implies the existence of a loop in the control policy. Now we argue this loop is Zeno.

First, by Remark 4.2, if $\tau_{q}=0$, then all trajectories spend zero time in $q$. Second, if $\tau_{q}>0$, then there exists $\bar{\tau}_{q}>0$ such that all trajectories spend at least $\bar{\tau}_{q}$ time in $q$. This follows from the boundedness of $f$ and the bisimulation construction (trajectories cannot move between two level sets of $\gamma_{n}^{\sigma}$ in arbitrarily small time). Since there is a finite number of states in $Q$, there exists $\bar{\tau}>0$, the minimum time spent by any trajectory in a state $q \in Q$ with $\tau_{q}>0$. The result is that Zeno trajectories only arise by an infinite number of control switches in a zero duration time interval. Hence, we have shown that the loop consists of control switches and/or zero duration time steps only, i.e., it is a Zeno loop.

Example 5.1. Consider the automaton in Figure 5.2. Suppose that we define a control policy $c\left(q_{1}\right)=\sigma^{\prime}, c\left(q_{3}\right)=\sigma^{\prime}, c\left(q_{4}\right)=\sigma$, and $c\left(q_{5}\right)=\sigma$. Starting at $q_{1}$ two possible trajectories are $q_{1} \stackrel{\sigma^{\prime}}{\rightarrow} q_{3} \stackrel{\sigma^{\prime}}{\rightarrow} q_{4} \stackrel{\sigma}{\rightarrow} q_{2}$, or $q_{1} \stackrel{\sigma^{\prime}}{\rightarrow} q_{3} \stackrel{\sigma^{\prime}}{\rightarrow} q_{4} \stackrel{\sigma}{\rightarrow} q_{1}$. The first trajectory has a zero duration time step. The control is inadmissible since the second trajectory has a Zeno loop.

5.2. Dynamic programming. We formulate the dynamic programming problem on $A$. This involves defining a cost-to-go function and a value function that minimizes it over control policies suitable for nondeterministic automata.

Let $\pi=q_{0} \stackrel{\sigma_{1}}{\rightarrow} q_{1} \rightarrow \cdots \rightarrow q_{N-1} \stackrel{\sigma_{N}}{\rightarrow} q_{N}$, where $q_{i}=\left[\left(\sigma_{i}, \xi_{i}\right)\right]$ and $\pi$ takes the sequence of edges $e_{1} e_{2} \ldots e_{N}$. We define a discrete cost-to-go $\hat{J}: Q \times \mathcal{C} \rightarrow \mathbb{R}$ by

$$
\hat{J}(q, c)= \begin{cases}\max _{\pi \in \tilde{\Pi}_{c}(q)}\left\{\sum_{j=1}^{N_{\pi}} \hat{L}\left(e_{j}\right)+\hat{h}\left(q_{N_{\pi}}\right)\right\} & \text { if } \Pi_{c}(q)=\tilde{\Pi}_{c}(q), \\ \infty & \text { otherwise }\end{cases}
$$

where $N_{\pi}=\min \left\{j \geq 0 \mid q_{j} \in Q_{f}\right\}$. We take the maximum over $\tilde{\Pi}_{c}(q)$ because of the nondeterminacy of $A$ : it is uncertain which among the (multiple) trajectories allowed by $c$ will be taken so we must assume the worst-case situation. The discrete value function $\hat{V}: Q \rightarrow \mathbb{R}$ is

$$
\hat{V}(q)=\min _{c \in \mathcal{C}} \hat{J}(q, c)
$$

for $q \in Q \backslash Q_{f}$ and $\hat{V}(q)=\hat{h}(q)$ for $q \in Q_{f}$. We show in Proposition 5.2 that $\hat{V}$ satisfies a DPP that takes into account the nondeterminacy of $A$ and ensures that optimal control policies are admissible. Let $\mathcal{A}_{q}$ be the set of control assignments $c(q) \in \Sigma_{\delta}$ at $q$ such that $c$ is admissible. 
Proposition 5.2. $\hat{V}$ satisfies

$$
\begin{array}{ll}
\hat{V}(q)=\min _{c(q) \in \mathcal{A}_{q}}\left\{\max _{e=\left(q, q^{\prime}\right) \in E_{c(q)}(q)}\left\{\hat{L}(e)+\hat{V}\left(q^{\prime}\right)\right\}\right\}, & q \in Q \backslash Q_{f}, \\
\hat{V}(q)=\hat{h}(q), & q \in Q_{f} .
\end{array}
$$

Proof. Fix $q \in Q$. By definition of $\hat{J}$,

$$
\hat{J}(q, c)=\max _{e=\left(q, q^{\prime}\right) \in E_{c(q)}(q)}\left\{\hat{L}(e)+\hat{J}\left(q^{\prime}, c\right)\right\} .
$$

By definition of $\hat{V}$,

$$
\hat{J}(q, c) \geq \max _{e=\left(q, q^{\prime}\right) \in E_{c(q)}(q)}\left\{\hat{L}(e)+\hat{V}\left(q^{\prime}\right)\right\} .
$$

Since $c(q) \in \mathcal{A}_{q}$ is arbitrary,

$$
\hat{V}(q) \geq \min _{c(q) \in \mathcal{A}_{q}}\left\{\max _{e=\left(q, q^{\prime}\right) \in E_{c(q)}(q)}\left\{\hat{L}(e)+\hat{V}\left(q^{\prime}\right)\right\}\right\} .
$$

To prove the reverse inequality suppose, by way of contradiction, there exists $\sigma^{\prime} \in \Sigma_{\delta}$ such that

$$
\hat{V}(q)>\max _{e=\left(q, q^{\prime}\right) \in E_{\sigma^{\prime}}(q)}\left\{\hat{L}(e)+\hat{V}\left(q^{\prime}\right)\right\}:=\hat{L}(e)+\hat{V}(\bar{q}) .
$$

Suppose an optimal admissible policy for $\bar{q}$ is $\bar{c}$. Define $c=\bar{c}$ on $Q \backslash\{q\}$ and $c(q)=\sigma^{\prime}$. Then $\hat{J}(q, c)=\hat{L}(e)+\hat{V}(\bar{q})<\hat{V}(q)$. This gives rise to a contradiction if we can show $c$ is admissible. Suppose not. Then there exists a loop of control switches and zero duration time steps containing $q$. Either the loop includes $\bar{q}$, implying $\hat{V}(\bar{q})=\hat{V}(q)$, which contradicts hypothesis (5.7), or the loop includes some other $q^{\prime}$ such that $\left(q, q^{\prime}\right) \in E_{\sigma^{\prime}}(q)$, implying $\hat{V}\left(q^{\prime}\right)=\hat{V}(q)$. But $\hat{V}(\bar{q}) \geq \hat{V}\left(q^{\prime}\right)$ since $\bar{q}$ gives the worst-case cost over edges with label $\sigma^{\prime}$. This again contradicts hypothesis (5.7).

5.3. Synthesis of $\boldsymbol{g}_{\boldsymbol{e}}$. The synthesis of enabling conditions or hybrid controller synthesis is typically a postprocessing step of a backward reachability analysis (see, for example, [52]). This situation prevails here as well: (5.4) and (5.5) describe a backward analysis to construct an optimal policy $c \in \mathcal{C}$. Once $c$ is known, the enabling conditions of $H$ are extracted as follows.

Consider each $e=\left(\sigma, \sigma^{\prime}\right) \in E$ of $H$ with $\sigma \neq \sigma^{\prime}$. There are two cases. If $\sigma^{\prime} \neq \sigma_{f}$, then $g_{e}=\left\{x \mid(\sigma, x) \in q, q \in Q, c(q)=\sigma^{\prime}\right\}$. That is, if the control policy designates switching from $q \in Q$ with label $\sigma$ to $q^{\prime} \in Q$ with label $\sigma^{\prime}$, then the corresponding enabling condition in $H$ includes the projection to $\mathbb{R}^{n}$ of $q$. The second case when $\sigma^{\prime}=\sigma_{f}$ is for edges going to the terminal location of $H$. Then $g_{e}=\{x \mid(\sigma, x) \in$ $\left.q, q \in Q_{f}\right\}$.

6. Main result. We will prove that $\hat{V}$ converges to $V$, the viscosity solution of the HJB equation, as $\delta_{Q}, \delta \rightarrow 0$. We make use of a filtration of control sets $\Sigma_{k} \equiv \Sigma_{\delta_{k}}$ corresponding to a sequence $\delta_{k} \rightarrow 0$ as $k \rightarrow \infty$ in such a manner that $\Sigma_{k} \subset \Sigma_{k+1}$. Considering (4.2), we define a filtration of families of submanifolds such that $\mathcal{W}_{k}^{\sigma} \subset \mathcal{W}_{k+1}^{\sigma}$ for each $\sigma \in \Sigma_{k}$. 
The proof proceeds in three steps. In the first step we restrict the class of controls to piecewise constant functions whose constant intervals are a function of the state. In particular, the control is constant on equivalence classes of $\simeq$. As $\delta_{k}$ tends to zero this class of piecewise constant controls well approximates $\epsilon$-optimal controls. The ArzelaAscoli theorem is invoked to show that the limit of a sequence of approximations $V_{k}$ of the value function using the aforementioned controls is a continuous function $V_{*}$. Using techniques of [4], $V_{*}$ is shown to be the unique viscosity solution of HJB. In the second step we introduce the discrete approximations of $L$ and $h$. The discrete approximation of $h$ is a one-time error, while the error between $L$ and $\hat{L}$ is shown to be $O\left(\delta_{k}^{2}\right)$ per interval $\tau$. Since the number of intervals is $O\left(1 / \delta_{k}\right)$, the error is $O\left(\delta_{k}\right)$. In the last step we introduce the discrete states $Q$. The error introduced at each control switch by the nondeterminacy of $A$ is $O\left(\delta_{k}\right)$ and since there are a fixed number of control switches as $\delta_{k} \rightarrow 0$, this error can be made arbitrarily small.

Step 1: Piecewise constant controls. In the first step we define a class of piecewise constant functions that depend on the state and show that the value function which minimizes the cost-to-go over this class converges to the viscosity solution of HJB as $\delta_{k} \rightarrow 0$. The techniques of this step are based on those by Bardi and Capuzzo-Dolcetta [4] and are related to those in [12].

We consider the optimal control problem (2.2)-(2.4) when the set of admissible controls is $\mathcal{U}_{k}^{1}$, piecewise constant functions consisting of finite sequences of control events $\sigma \in \Sigma_{k}$, where each $\sigma$ is applied for a time $\tau(\sigma, x)$ and the trajectory remains in $\Omega$. Let $(\sigma, x) \in q$ for $q \in Q$ and define $\tau(\sigma, x)$ to be the minimum of the time it takes the trajectory starting at $x$ and using control $\sigma \in \Sigma_{k}$ to reach (ta) $\partial \Omega_{f}$ or (tb) some $x^{\prime}$ such that $\left(\sigma, x^{\prime}\right) \notin q$. If a trajectory is at $x_{i}$ at the start of the $(i+1)$ th step, then the control $\sigma_{i+1}$ is applied for time $\tau_{i+1}:=\tau\left(\sigma_{i+1}, x_{i}\right)$ and $x_{i+1}=\phi_{\tau_{i+1}}\left(x_{i}, \sigma_{i+1}\right)$. Thus $U_{k}^{1}$ is a class of piecewise constant controls whose constant intervals are based on the state partition induced by $\simeq$ (in contrast with a partition of the time interval): the control can only change values on the boundary of equivalence classes.

Let

$$
\mathcal{R}_{k}^{1}:=\left\{x \in \Omega \mid \exists \mu \in \mathcal{U}_{k}^{1} \cdot T(x, \mu)<\infty\right\} .
$$

We define the cost-to-go function $J_{k}^{1}: \Omega \times \mathcal{U}_{k}^{1} \rightarrow \mathbb{R}$ as follows. For $x \in \Omega$ and $\mu=\sigma_{1} \sigma_{2} \ldots \in \mathcal{U}_{k}^{1}$, if $T(x, \mu)<\infty$, then

$$
J_{k}^{1}(x, \mu)=\sum_{j=1}^{N} \int_{0}^{\tau\left(\sigma_{j}, x_{j-1}\right)} L\left(\phi_{s}\left(x_{j-1}, \sigma_{j}\right), \sigma_{j}\right) d s+h\left(x_{N}\right),
$$

where $N=\min \left\{j \geq 0 \mid x_{j} \in \Omega_{f}\right\} . J_{k}^{1}(x, \mu)=\infty$ otherwise. We define the value function $V_{k}^{1}: \mathbb{R}^{n} \rightarrow \mathbb{R}$ as follows. For $x \in \Omega \backslash \Omega_{f}$,

$$
V_{k}^{1}(x)=\inf _{\mu \in \mathcal{U}_{k}^{1}} J_{k}^{1}(x, \mu),
$$

and for $x \in \Omega_{f}, V_{k}^{1}(x)=h(x)$. The following result is proved using standard arguments from dynamic programming [20].

Proposition 6.1. $V_{k}^{1}$ satisfies, for all $x \in \mathcal{R}_{k}^{1}$,

$$
V_{k}^{1}(x)=\min _{\sigma \in \Sigma_{k}}\left\{\int_{0}^{\tau(\sigma, x)} L\left(\phi_{s}(x, \sigma), \sigma\right) d s+V_{k}^{1}\left(\phi_{\tau(\sigma, x)}(x, \sigma)\right)\right\} .
$$




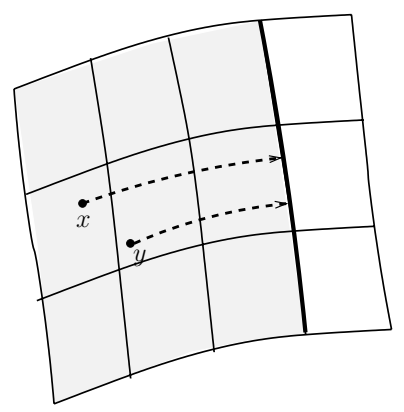

FIG. 6.1. The shaded region is $M_{c-}^{\sigma}$ while the bold curve on its boundary is $M_{c}^{\sigma}$.

We would like to show that $V_{k}^{1}$ is uniformly bounded and locally uniformly continuous. Considering uniform continuity of $V_{k}^{1}$, let $C_{k}$ be as in (4.1) and $\gamma_{n}^{\sigma}$ the submersion whose level sets are transverse to the flow of $\dot{x}=f(x, \sigma)$. Referring to Figure 6.1, for each $\sigma \in \Sigma_{k}$ and for each fixed $c \in C_{k}$ we define the regions in $\mathbb{R}^{n}$

$$
\begin{aligned}
M_{c}^{\sigma} & :=\left\{x \mid \gamma_{n}^{\sigma}(x)=c\right\}, \\
M_{c-}^{\sigma}: & :=\left\{x \mid \gamma_{n}^{\sigma}(x) \in(-1, c)\right\} ;
\end{aligned}
$$

that is, $M_{c-}$ is the strip of points belonging to a level set of $\gamma_{n}^{\sigma}$ whose level value is between -1 and $c$.

Remark 6.1.

(a) If $x, y \in M_{c-}^{\sigma}$ for some $c \in C_{k}$ and $\tau(\sigma, x)$ and $\tau(\sigma, y)$ are defined using (tb), then $|\tau(\sigma, x)-\tau(\sigma, y)| \rightarrow 0$ and $\left\|\phi_{\tau(\sigma, x)}(x, \sigma)-\phi_{\tau(\sigma, y)}(y, \sigma)\right\| \rightarrow 0$ as $\|x-y\| \rightarrow 0$ in $M_{c-}^{\sigma}$, since $M_{c}^{\sigma}$ is a smooth submanifold. See Figure 6.1. For the details, see [20, Theorem 6.1, pp. 91-94]. If instead $\tau(\sigma, x)$ and $\tau(\sigma, y)$ are defined using (ta) and $\sigma$ is an $\epsilon$-optimal control for $x$, then by Assumption 2.2 the same results hold.

(b) For each $x \in \cup_{k} \mathcal{R}_{k}^{1}$ and $\epsilon>0$ there exists $m \in \mathbb{Z}^{+}$and $\mu \in \mathcal{U}_{m}^{1}$ such that $\mu$ is an $\epsilon$-optimal control for $x$ w.r.t. $V_{k}^{1}$ with at most $N_{\epsilon}$ discontinuities and such that $\phi_{t}(x, \mu)$ is transverse to $\partial \Omega_{f}$. This follows from Assumption 2.2, $V_{k}^{1}(x) \geq V(x)$, and the fact that we can well approximate an $\epsilon$-optimal control for $V$ by a control in $\mathcal{U}_{m}^{1}$ for large enough $m$.

The following lemma shows that $V_{k}^{1}$ is locally uniformly continuous.

Lemma 6.2. For each $y \in \cup_{k} \mathcal{R}_{k}^{1}$ and $\epsilon>0$, there exists $m_{\epsilon} \in \mathbb{Z}^{+}$and $\eta_{\epsilon}>0$ such that $\left|V_{k}^{1}(x)-V_{k}^{1}(y)\right|<2 \epsilon$ for all $|x-y|<\eta_{\epsilon}$ with $x \in \mathcal{R}_{k}^{1}$ and for all $k>m_{\epsilon}$.

Proof. Fix $y \in \cup_{k} \mathcal{R}_{k}^{1}$. By Remark 6.1(b) there exists $m_{1}>0$ and $\mu \in \mathcal{U}_{m_{1}}^{1}$ such that $\mu$ is an $\epsilon$-optimal control for $y$ satisfying Assumption 2.2. Let $x \in \mathcal{R}_{m_{1}}^{1}$. Then $V_{k}^{1}(x)-V_{k}^{1}(y) \leq J_{k}^{1}\left(x, \mu_{x}\right)-J_{k}^{1}(y, \mu)+\epsilon$ for any $\mu_{x} \in \mathcal{U}_{m_{1}}^{1}$ and $k>m_{1}$. If we can show that for fixed $y$ and $\mu$ there exists $\mu_{x} \in \mathcal{U}_{m_{1}}^{1}$ such that

$$
J_{k}^{1}\left(x, \mu_{x}\right)-J_{k}^{1}(y, \mu)<\epsilon
$$

for all $x \in \mathcal{R}_{m_{1}}^{1}$ sufficiently close to $y$, then $V_{k}^{1}(x)-V_{k}^{1}(y) \leq 2 \epsilon$ for all $k \geq m_{1}$.

Conversely, by Remark 6.1(b) there exists $m_{2}>0$ and $\mu_{x} \in \mathcal{U}_{m_{2}}^{1}$ such that $\mu_{x}$ is an $\epsilon$-optimal control for $x$ satisfying Assumptions 2.2. Then $V_{k}^{1}(y)-V_{k}^{1}(x) \leq$ $J_{k}^{1}(y, \mu)-J_{k}^{1}\left(x, \mu_{x}\right)+\epsilon$ for any $\mu \in \mathcal{U}_{m_{2}}^{1}$ and $k>m_{2}$. If we can show that for fixed $y$ there exists $\mu \in \mathcal{U}_{m_{2}}^{1}$ such that

$$
J_{k}^{1}(y, \mu)-J_{k}^{1}\left(x, \mu_{x}\right)<\epsilon
$$


for all $x \in \mathcal{R}_{m_{2}}^{1}$ sufficiently close to $y$, then $V_{k}^{1}(x)-V_{k}^{1}(y) \geq-2 \epsilon$ for all $k \geq m_{2}$. The result follows by letting $m_{\epsilon}=\min \left\{m_{1}, m_{2}\right\}$. Thus, we must show (6.3) and (6.4).

Consider first (6.3). Let $\mu=\sigma_{1} \sigma_{2} \ldots \in \mathcal{U}_{k}^{1}$ be an $\epsilon$-optimal control for $y$ such that $y_{N} \in \partial \Omega_{f}$. By redefining indices, we can associate with $\mu$ the open-loop control $\tilde{\mu}=\left(\sigma_{1}, \tau_{1}\right)\left(\sigma_{2}, \tau_{2}\right) \ldots$, where $\tau_{i}$ is the time $\sigma_{i}$ is applied. We claim there exists $\tilde{\mu}^{x}=\left(\sigma_{1}, \tau_{1}^{x}\right)\left(\sigma_{2}, \tau_{2}^{x}\right) \ldots$ such that as $x \rightarrow y$, (a) $x_{j} \rightarrow y_{j}$, (b) $\tau_{j}^{x} \rightarrow \tau_{j}$, and (c) $x_{N} \in \partial \Omega_{f}$. Let $T_{k}=\max _{i} \tau_{i}$. Then we have

$$
\begin{aligned}
J_{k}^{1}\left(x, \tilde{\mu}^{x}\right)-J_{k}^{1}(y, \tilde{\mu}) \leq & \sum_{j=1}^{N} \int_{0}^{\tau_{j}}\left|L\left(\phi_{s}\left(x_{j-1}, \sigma_{j}\right), \sigma_{j}\right)-L\left(\phi_{s}\left(y_{j-1}, \sigma_{j}\right), \sigma_{j}\right)\right| d s \\
& +\sum_{j=1}^{N}\left|\int_{\tau_{j}}^{\tau_{j}^{x}} L\left(\phi_{s}\left(x_{j-1}, \sigma_{j}\right), \sigma_{j}\right) d s\right|+\left|h\left(y_{N}\right)-h\left(x_{N}\right)\right| \\
\leq & L_{L} T_{k} \exp \left(L_{f} T_{k}\right) \sum_{j=1}^{N}|| x_{j-1}-y_{j-1} \| \\
& +M_{L} \sum_{j=1}^{N}\left|\tau_{j}^{x}-\tau_{j}\right|+L_{h}\left|x_{N}-y_{N}\right| .
\end{aligned}
$$

By the previous claim the right-hand side (r.h.s.) can be made less than $\epsilon$. Thus, we need only show there exists $\tilde{\mu}^{x}=\left(\sigma_{1}, \tau_{1}^{x}\right)\left(\sigma_{2}, \tau_{2}^{x}\right) \ldots$, which satisfies the claim, and $\mu^{x} \in \mathcal{U}_{k}^{1}$ can be reconstructed from it, based on the discrete states in $Q$ visited by $\phi_{t}\left(x, \tilde{\mu}^{x}\right)$.

We argue by induction. Suppose (a)-(c) hold at $j-1$. We show they hold at $j$. We need only consider the case when $y_{j-1} \in M_{c-}^{\sigma_{j}}$ and $y_{j} \in M_{c}^{\sigma_{j}}$ for some $c \in C_{k}$; that is, $y_{j-1}$ lies upstream of $y_{j}$ (trajectories flow in the increasing $\gamma_{n}^{\sigma_{j}}$ direction), while $y_{j}$ lies on the boundary of an equivalence class where the control is allowed to switch values. For $x_{j-1}$ sufficiently close to $y_{j-1}, x_{j-1} \in M_{c-}^{\sigma_{j}}$. By Remark 6.1(a) there exists $\tau_{j}^{x}$ such that $x_{j}=\phi_{\tau_{j}^{x}}\left(x_{j-1}, \sigma_{j}\right) \in M_{c}^{\sigma_{j}}$ and $\tau_{j}^{x} \rightarrow \tau_{j}$ and $x_{j} \rightarrow y_{j}$ as $x_{j-1} \rightarrow y_{j-1}$. The case $y_{j-1} \in M_{c-}^{\sigma_{j}}$ and $y_{j} \in \partial \Omega_{f}$ follows in the same way from Assumption 2.2. Proving (6.4) follows along the same lines as the proof for (6.3).

To show boundedness of $V_{k}^{1}$, let $T(x):=\inf _{\mu \in \mathcal{U}_{k}^{1}} T(x, \mu)$. In light of Assumption 2.1(2), we have that for all $x \in \mathbb{R}^{n},\left|V_{k}^{1}(x)\right| \leq T(x) \cdot M_{L}+M_{h}$. Consider the set $K_{a}:=\left\{x \in \mathcal{R}_{k}^{1} \mid T(x)<a\right\}$. Then $\left|V_{k}^{1}(x)\right| \leq a \cdot M_{L}+M_{h}$ for all $x \in K_{a}$.

We have shown that on each $K_{a} \subseteq \mathbb{R}^{n},\left\{V_{k}^{1}\right\}$ forms a family of equibounded, locally equicontinuous functions. It follows by the Arzela-Ascoli theorem [42] that along some subsequence $k_{n}, V_{k_{n}}^{1}$ converges to a continuous function $V_{*}$. The proof of the following result closely follows [4].

Proposition 6.3. $V_{*}$ is the unique viscosity solution of HJB.

Proof. We show that $V_{*}$ solves HJB in the viscosity sense. Let $\psi \in C^{1}\left(\mathbb{R}^{n}\right)$ and suppose $x_{0} \in \Omega$ is a strict local maximum for $V_{*}-\psi$. There exists a closed ball $B$ centered at $x_{0}$ such that $\left(V_{*}-\psi\right)\left(x_{0}\right)>\left(V_{*}-\psi\right)(x)$ for all $x \in B$. Let $x_{0 \delta_{k}}$ be a maximum point for $V_{k}^{1}-\psi$ over $B$. Since $V_{k}^{1} \rightarrow V_{*}$ locally uniformly it follows that $x_{0 \delta_{k}} \rightarrow x_{0}$ as $\delta_{k} \rightarrow 0$. Then, for any $\sigma \in \Sigma_{k}$, the point $\phi_{\tau}\left(x_{0 \delta_{k}}, \sigma\right)$ is in $B$ (using boundedness of $f$ ), for sufficiently small $\delta_{k}$ and $0 \leq \tau \leq \tau\left(x_{0 \delta_{k}}, \sigma\right)$, since $\tau\left(x_{0 \delta_{k}}, \sigma\right) \rightarrow 0$ as $\delta_{k} \rightarrow 0$. Therefore,

$$
V_{k}^{1}\left(x_{0 \delta_{k}}\right)-\psi\left(x_{0 \delta_{k}}\right) \geq V_{k}^{1}\left(\phi_{\tau}\left(x_{0 \delta_{k}}, \sigma\right)\right)-\psi\left(\phi_{\tau}\left(x_{0 \delta_{k}}, \sigma\right)\right) .
$$


Considering (6.2), we have

$$
\begin{aligned}
0 & =-\min _{\sigma \in \Sigma_{k}}\left\{V_{k}^{1}\left(\phi_{\tau}\left(x_{0 \delta_{k}}, \sigma\right)\right)-V_{k}^{1}\left(x_{0 \delta_{k}}\right)+\int_{0}^{\tau} L\left(\phi_{s}\left(x_{0 \delta_{k}}, \sigma\right), \sigma\right) d s\right\} \\
& \geq-\min _{\sigma \in \Sigma_{k}}\left\{\psi\left(\phi_{\tau}\left(x_{0 \delta_{k}}, \sigma\right)\right)-\psi\left(x_{0 \delta_{k}}\right)+\int_{0}^{\tau} L\left(\phi_{s}\left(x_{0 \delta_{k}}, \sigma\right), \sigma\right) d s\right\} .
\end{aligned}
$$

Since $\psi \in C^{1}\left(\mathbb{R}^{n}\right)$, we have by the mean value theorem,

$$
0 \geq-\min _{\sigma \in \Sigma_{k}}\left\{\frac{\partial \psi}{\partial x}(y) \cdot \int_{0}^{\tau} f\left(\phi_{s}\left(x_{0 \delta_{k}}, \sigma\right), \sigma\right) d s+\int_{0}^{\tau} L\left(\phi_{s}\left(x_{0 \delta_{k}}, \sigma\right), \sigma\right) d s\right\},
$$

where $y=\alpha x_{0 \delta_{k}}+(1-\alpha) \phi_{\tau}\left(x_{0 \delta_{k}}, \sigma\right)$ for some $\alpha \in(0,1)$. Dividing by $\tau>0$ on each side and taking the limit as $\delta_{k} \rightarrow 0$, we have $V_{k}^{1} \rightarrow V_{*}, x_{0 \delta_{k}} \rightarrow x_{0}, \tau \rightarrow 0$, and $y \rightarrow x_{0 \delta_{k}}$. By the fundamental theorem of calculus, the continuity of $f$ and $L$, and the uniform continuity in $u$ of the expression in brackets, we obtain

$$
0 \geq-\inf _{u \in U}\left\{\frac{\partial \psi}{\partial x}\left(x_{0}\right) \cdot f\left(x_{0}, u\right)+L\left(x_{0}, u\right)\right\} .
$$

This confirms part (i) of the viscosity solution definition. Part (ii) is proved in an analogous manner.

Step 2: Approximate cost functions and overapproximation of $\Omega_{f}$ by $Q_{f}$. In this step we define a class of piecewise constant controls, denoted $\mathcal{U}_{k}^{2}$, nearly the same as $\mathcal{U}_{k}^{1}$, to accommodate that trajectories terminate at $Q_{f}$, not $\Omega_{f}$, and we replace the cost functions $L$ and $h$ by approximations $L^{2}$ and $\hat{h}$, respectively. We define $\mathcal{U}_{k}^{2} \subset \mathcal{U}_{k}^{1}$ to be the class of piecewise continuous controls whose constant time intervals $\tau(\sigma, x)$ are determined by the equivalence classes of $\simeq$ but not $\partial \Omega_{f}$. That is, case (ta) in the definition of $\tau(\sigma, x)$ in Step 1 is omitted. Next we define an approximate instantaneous cost $L^{2}: \Omega \times \Sigma_{k} \rightarrow \mathbb{R}$ by

$$
L^{2}(x, \sigma):=\hat{L}(e),
$$

where $(\sigma, x) \in q$ and $e=\left(q, q^{\prime}\right)$ represents the time step. For $x \in \Omega$ and $\mu=\sigma_{1} \sigma_{2} \ldots \in$ $\mathcal{U}_{k}^{2}$, if $T(x, \mu)<\infty$, the cost-to-go function $J_{k}^{2}: \Omega \times \mathcal{U}_{k}^{2} \rightarrow \mathbb{R}$ is

$$
J_{k}^{2}(x, \mu)=\sum_{j=1}^{N} L^{2}\left(x_{j-1}, \sigma_{j}\right)+\hat{h}\left(x_{N}\right),
$$

where $N=\min \left\{j \geq 0 \mid x_{j} \in Q_{f}\right\}$. In other words, $J_{k}^{2}$ is a worst-case cost over a set of trajectories starting at $x$ that visit the same sequence of equivalence classes of $\simeq$, and it is a worst-case cost w.r.t. $J_{k}^{1}$ because $\int_{0}^{\tau(\sigma, x)} L\left(\phi_{s}(x, \sigma), \sigma\right) d s \leq L^{2}(x, \sigma)$.

We define a value function $V_{k}^{2}: \mathbb{R}^{n} \rightarrow \mathbb{R}$ as follows. For $x \in \Omega \backslash Q_{f}$,

$$
V_{k}^{2}(x)=\inf _{\mu \in \mathcal{U}_{k}^{1}} J_{k}^{2}(x, \mu),
$$

and for $x \in Q_{f}, V_{k}^{2}(x)=\hat{h}(x)$. For $x \in \Omega$ such that $V_{k}^{2}(x)<\infty, V_{k}^{2}$ satisfies the DPP

$$
V_{k}^{2}(x)=\min _{\sigma \in \Sigma_{k}}\left\{L^{2}(x, \sigma)+V_{k}^{2}\left(\phi_{\tau(\sigma, x)}(x, \sigma)\right)\right\} .
$$

The proof is along the same lines as that of Proposition 5.2. 
The following facts are useful for the subsequent result. The first lemma says that $\tau_{q}$ is order $\delta_{k}$. The second lemma says that given two times $\tau$ and $\tau^{\prime}$ that two trajectories spend, respectively, in the same equivalence class, $\left|\tau-\tau^{\prime}\right|$ is order $\delta_{k}^{2}$.

LEMMA 6.4. If $\delta_{k}<\frac{m_{f}}{L_{f}}$, then for all $q \in Q$,

$$
\tau_{q} \leq \frac{\delta_{k}}{m_{f}-L_{f} \delta_{k}} .
$$

Proof. Let $q \in Q$. Fix $x \in \Omega$ and $\sigma \in \Sigma_{k}$ such that $(\sigma, x) \in q$. We know $\left\|\phi_{\tau(\sigma, x)}(x, \sigma)-x\right\| \leq \delta_{k}$. We have

$$
\begin{aligned}
\delta_{k} \geq\left\|\phi_{\tau(\sigma, x)}-x\right\| & =\left\|\int_{0}^{\tau(\sigma, x)} f\left(\phi_{s}(x, \sigma), \sigma\right) d s\right\| \\
& \geq\left\|\int_{0}^{\tau(\sigma, x)} f(x, \sigma) d s\right\|-\left\|\int_{0}^{\tau(\sigma, x)}\left[f\left(\phi_{s}(x, \sigma), \sigma\right)-f(x, \sigma)\right] d s\right\| \\
& \geq \tau(\sigma, x)\|f(x, \sigma)\|-\tau(\sigma, x) L_{f} \delta_{k},
\end{aligned}
$$

where in the last step we use the fact that $\left\|\phi_{s}(x, \sigma)-x\right\| \leq \delta_{k}$. Therefore,

$$
\tau(\sigma, x) \leq \frac{\delta_{k}}{\|f(x, \sigma)\|-L_{f} \delta_{k}} .
$$

Using Assumption 4.1(2) and taking the sup over all $\tau(\sigma, x)$ for $q$, the result follows.

Lemma 6.5. Let $x, x^{\prime} \in M_{c}^{\sigma}$ for some $c \in C_{k}$ and $\sigma \in \Sigma_{k}$ such that $\left\|x-x^{\prime}\right\| \leq \delta_{k}$. Let $\tau, \tau^{\prime}$ be times such that $\phi_{\tau}(x, \sigma), \phi_{\tau^{\prime}}\left(x^{\prime}, \sigma\right) \in M_{c+\Delta}^{\sigma}$. Then $\left|\tau-\tau^{\prime}\right| \leq c_{\gamma} \tau \delta_{k}$ for some $c_{\gamma}>0$.

Proof. We have

$$
\int_{0}^{\tau} \frac{d}{d s}\left(\gamma_{n}^{\sigma}\left(\phi_{s}(x, \sigma)\right)\right) d s=\int_{0}^{\tau^{\prime}} \frac{d}{d s}\left(\gamma_{n}^{\sigma}\left(\phi_{s}\left(x^{\prime}, \sigma\right)\right)\right) d s
$$

Let $f=f\left(\phi_{s}(x, \sigma), \sigma\right), f^{\prime}=f\left(\phi_{s}\left(x^{\prime}, \sigma\right), \sigma\right), d \gamma=\left.\frac{d \gamma_{n}^{\sigma}(z)}{d z}\right|_{z=\phi_{s}(x, \sigma)}$, and $d \gamma^{\prime}=$ $\left.\frac{d \gamma_{n}^{\sigma}(z)}{d z}\right|_{z=\phi_{s}\left(x^{\prime}, \sigma\right)}$. Then, rearranging terms,

$$
\int_{0}^{\tau}\left(f^{\prime} \cdot d \gamma^{\prime}\right) d s-\int_{0}^{\tau}(f \cdot d \gamma) d s=\int_{\tau^{\prime}}^{\tau}\left(f^{\prime} \cdot d \gamma^{\prime}\right) d s .
$$

Let $L_{1}$ be the Lipschitz constant of $f \cdot d \gamma$ (using the fact that $\gamma_{n}^{\sigma}$ is smooth). Then

$$
\int_{\tau^{\prime}}^{\tau} f^{\prime} \cdot d \gamma^{\prime} \leq L_{1} \tau\left\|x-x^{\prime}\right\| \leq L_{1} \tau \delta_{k}
$$

Since $\gamma_{n}^{\sigma}$ defines a transversal foliation to vector field $f(\cdot, \sigma), f \cdot d \gamma>0$. Let $c=$ $\min _{s \in\left[\tau, \tau^{\prime}\right]}\left\{f^{\prime} \cdot d \gamma^{\prime}\right\}>0$. Letting $c_{\gamma}=\frac{L_{1}}{c}$ we obtain the result.

Remark 6.2. If $\mu \in \mathcal{U}_{k}^{1}$ is an $\epsilon$-optimal control for $x$ and the first time the trajectory $\phi_{t}(x, \mu)$ reaches $\Omega_{f}\left(Q_{f}\right)$ is $T\left(T^{2}\right)$, then $T-T^{2} \rightarrow 0$ and $\mid \phi_{T}(x, \mu)-$ $\phi_{T^{2}}(x, \mu) \mid \rightarrow 0$ as $k \rightarrow \infty$. This follows from the fact that the distance between $\Omega_{f}$ and $Q_{f}$ tends to zero as $k \rightarrow \infty$. 
We denote by $\mu^{2} \in \mathcal{U}_{k}^{2}$ the restriction of $\mu$ to $\left[0, T^{2}\right]$. Note that if the length of $\mu$ is $|\mu|=N$, then $\left|\mu_{2}\right|:=N^{2} \leq N$. Then we have the following result.

Proposition 6.6. Let $k_{0} \in \mathbb{Z}^{+}$be arbitrary, $x \in \mathcal{R}_{k_{0}}^{1}$, and $\mu \in \mathcal{U}_{k_{0}}^{1}$ be an $\epsilon$-optimal control for $x$. Then $\left|J_{k}^{1}(x, \mu)-J_{k}^{2}\left(x, \mu^{2}\right)\right| \rightarrow 0$ as $k \rightarrow \infty$.

Proof. Suppose $\mu=\left(\sigma_{1}, \tau_{1}\right) \ldots\left(\sigma_{N}, \tau_{N}\right)$ and $\mu^{2}=\left(\sigma_{1}, \tau_{1}\right) \ldots\left(\sigma_{N^{2}}, \tau_{N^{2}}\right)$, where $N^{2} \leq N$. Thus, $N-N^{2}$ additional steps are required to reach $\partial \Omega_{f}$ after reaching $Q_{f}$. Then we have

$$
\begin{aligned}
\left|J_{k}^{1}(x, \mu)-J_{k}^{2}\left(x, \mu^{2}\right)\right| \leq & \mid \sum_{j=1}^{N}\left[\int_{0}^{\tau\left(\sigma_{j}, x_{j-1}\right)} L\left(\phi_{s}\left(x_{j-1}, \sigma_{j}\right), \sigma_{j}\right) d s\right]+h\left(x_{N}\right) \\
& -\sum_{j=1}^{N^{2}}\left[\tau_{q_{j-1}} L\left(\xi_{j-1}, \sigma_{j}\right)\right]-\hat{h}\left(x_{N^{2}}\right) \mid,
\end{aligned}
$$

where $\left(\sigma_{j}, x_{j-1}\right) \in q_{j-1}$ and $q_{j-1}=\left[\left(\sigma_{j}, \xi_{j-1}\right)\right]$. There exists $\xi_{N^{2}}$ such that $\hat{h}\left(x_{N^{2}}\right)=$ $h\left(\xi_{N^{2}}\right)$ and $\left\|x_{N^{2}}-\xi_{N^{2}}\right\| \leq \delta_{k}$. Also, using the mean value theorem, there exists $\tilde{t}_{j-1}$ with $\tilde{x}_{j-1}=\phi_{\tilde{t}_{j-1}}\left(x_{j-1}, \sigma_{j}\right)$ and $\left\|\tilde{x}_{j-1}-\xi_{j-1}\right\| \leq \delta_{k}$ such that

$$
\begin{aligned}
& \left|J_{k}^{1}(x, \mu)-J_{k}^{2}\left(x, \mu^{2}\right)\right| \\
& \leq \sum_{j=1}^{N^{2}}\left|\tau\left(\sigma_{j}, x_{j-1}\right) L\left(\tilde{x}_{j-1}, \sigma_{j}\right)-\tau_{q_{j-1}} L\left(\xi_{j-1}, \sigma_{j}\right)\right| \\
& \quad+\left|\sum_{j=N^{2}+1}^{N}\left[\int_{0}^{\tau\left(\sigma_{j}, x_{j-1}\right)} L\left(\phi_{s}\left(x_{j-1}, \sigma_{j}\right), \sigma_{j}\right) d s\right]\right|+\left|h\left(x_{N}\right)-\hat{h}\left(x_{N^{2}}\right)\right| \\
& \leq \sum_{j=1}^{N^{2}} \tau_{q_{j-1}} L_{L} \delta_{k}+\sum_{j=1}^{N^{2}}\left[\tau_{q_{j-1}}-\tau\left(\sigma_{j}, x_{j-1}\right)\right] L\left(\tilde{x}_{j-1}, \sigma_{j}\right) \\
& \quad+\left(T-T^{2}\right) M_{L}+L_{h}\left\|x_{N}-x_{N^{2}}\right\|+L_{h} \delta_{k} .
\end{aligned}
$$

The last three terms on the r.h.s. go to zero as $k \rightarrow \infty$ because of Remark 6.2 and since $\delta_{k} \rightarrow 0$. Using Lemma 6.4 the first summation decreases linearly as $\delta_{k}$. Call the second summation on the r.h.s. B. Splitting B into sums over control switches and time steps, we have

$$
\begin{aligned}
B \leq & M_{L} \sum_{j=1}^{N^{2}}\left[\tau_{q_{j-1}}-\tau\left(\sigma_{j}, x_{j-1}\right)\right] \mathbf{1}\left(\sigma_{j}=\sigma_{j-1}\right) \\
& +M_{L} \sum_{j=1}^{N^{2}}\left[\tau_{q_{j-1}}-\tau\left(\sigma_{j}, x_{j-1}\right)\right] \mathbf{1}\left(\sigma_{j} \neq \sigma_{j-1}\right) \\
\leq & M_{L} \sum_{j=1}^{N^{2}} c_{j-1} \tau_{q_{j-1}} \delta_{k}+M_{L} \sum_{j=1}^{N^{2}} \tau_{q_{j-1}} \mathbf{1}\left(\sigma_{j} \neq \sigma_{j-1}\right)
\end{aligned}
$$

for some $c_{j-1} \in \mathbb{R}$. In the second line we used Lemma 6.5 and the fact that $\tau_{q_{j-1}} \geq$ $\tau\left(\sigma_{j}, x_{j-1}\right)$. Using Lemma 6.4 the first summation on the r.h.s. decreases linearly as $\delta_{k}$. The second term on the r.h.s. goes to zero since, by Assumption 2.2, $\mu$ has a fixed number of control switches for all $k \geq k_{0}$. 
Step 3: Discrete states and nondeterminacy. In the last step we compare the value function $V_{k}^{2}(x)$ with the discrete value function $\hat{V}$ defined on $A$. The difference between the two is that trajectories defined over $\mathcal{U}_{k}^{2}$ do not include jumps, while trajectories whose time abstract versions are accepted by $A$ can have jumps due to the nondeterminacy of $A$. Nevertheless, as $k \rightarrow \infty$ this discrepancy can be made negligible and we show that the difference between $V_{k}^{2}$ and $\hat{V}$ can be made arbitrarily small.

First we extend the domain of $\hat{V}(q)$, with an abuse of notation, by defining

$$
\hat{V}_{k}(x):=\min _{\sigma \in \Sigma_{k}}\{\hat{V}(q) \mid(\sigma, x) \in q\} .
$$

Also let $\hat{\mathcal{R}}_{k}=\left\{x \in \Omega \mid \hat{V}_{k}(x)<\infty\right\}$ and $\hat{\mathcal{R}}=\cup_{k} \hat{\mathcal{R}}_{k}$.

Remark 6.3.

(a) For each $x \in \cup_{k} \mathcal{R}_{k}^{1}$ and $\epsilon>0$ there exists $m \in \mathbb{Z}^{+}$and $\mu \in \mathcal{U}_{m}^{2}$ such that $\mu$ is an $\epsilon$-optimal control for $x$ w.r.t. $V_{k}^{2}$ with at most $N_{\epsilon}$ discontinuities. This follows from Remark 6.1(b) and the fact that trajectories in $\mathcal{U}_{m}^{2}$ are merely truncations of trajectories in $\mathcal{U}_{m}^{1}$.

(b) $\hat{\mathcal{R}} \subset \cup_{k} \mathcal{R}_{k}^{1}$, but the converse is not true, in general.

(c) If $\mu$ is an $\epsilon$-optimal control for $x$ w.r.t. $V_{k}^{2}$, then we can assume $\phi_{t}(x, \mu)$ does not self-intersect, for if it did we could find $\tilde{\mu}$, also $\epsilon$-optimal, which eliminates loops in $\phi_{t}(x, \mu)$.

(d) $\left\|x-x^{\prime}\right\| \rightarrow 0$ as $k \rightarrow \infty$ for all $x, x^{\prime}, \sigma, \sigma^{\prime} \neq \sigma$ such that $\left([(\sigma, x)],\left[\left(\sigma^{\prime}, x^{\prime}\right)\right]\right) \in E$.

(e) For all $x \in \hat{R}, \hat{V}_{k}(x) \geq V_{k}^{2}(x)$. This follows because the argument of the max in the definition of $\hat{J}(q, c)$ is equal to $J_{k}^{2}(x, \mu)$, where $c$ is a control policy as defined in section 5.1 with $c(q)=\sigma_{1},\left(\sigma_{1}, x\right) \in q$, and $\mu=\left(\sigma_{1}, \tau_{1}\right) \ldots$ is the piecewise continuous control that corresponds to following policy $c$ starting at $x$. Thus, $J_{k}^{2}(x, \mu)$ is the cost for a particular trajectory in $\tilde{\Pi}_{c}(q)$ which has no jumps at the control switches. Then we have $\hat{J}(q, c) \geq J_{k}^{2}(x, \mu)$, since $\hat{J}(q, c)$ maximizes over all trajectories in $\tilde{\Pi}_{c}(q)$.

Proposition 6.7. For all $x \in \hat{\mathcal{R}},\left|\hat{V}_{k}(x)-V_{k}^{2}(x)\right| \rightarrow 0$ as $k \rightarrow \infty$.

Proof. Fix $\epsilon>0$ and $x \in \hat{\mathcal{R}}$. By Remark 6.3(a) there exists $m>0$ and an $\epsilon$-optimal control $\mu \in \mathcal{U}_{m}^{2}$ for $x$ w.r.t. $V_{m}^{2}$. Denote $\mu=\left(\left(\sigma_{1}, \tau_{1}\right) \ldots\left(\sigma_{N}, \tau_{N}\right)\right)$, where $\tau_{i}$ is the time $\sigma_{i}$ is applied. Let $c$ be any control policy on $Q$ that is generated using $\delta_{k}$ and $C_{k}$, for $k \geq m$. Then, using Remark 6.3(e),

$$
0 \leq \hat{V}_{k}(x)-V_{k}^{2}(x) \leq \hat{J}_{k}(q, c)-J_{k}^{2}(x, \mu)+\epsilon,
$$

where $q=\left[\left(\sigma_{1}, x\right)\right]$. If we can show there exists $\bar{k} \geq m$ such that for $k>\bar{k}$, there exists a policy $\bar{c}$ such that

$$
\hat{J}_{k}(q, \bar{c})-J_{k}^{2}(x, \mu)<\epsilon,
$$

then the result follows.

By Remark 6.3(d) and the transversality of $\phi_{t}(x, \mu)$ with the level sets of $\gamma_{n}$, we can find $\bar{k} \geq m$ such that for $k>\bar{k}$, there exists a family of (both continuous and discontinuous) trajectories $\Psi_{k}$ starting at $x$ with the following properties:

(1) $\phi_{t}(x, \mu) \in \Psi_{k}$.

(2) $\phi \in \Psi_{k}$ is defined over a control $\tilde{\mu}=\left(\left(\sigma_{1}, \tilde{\tau}_{1}\right), \ldots,\left(\sigma_{N}, \tilde{\tau}_{N}\right)\right) \in \mathcal{U}_{k}^{2}$ with the same sequence of control values as $\mu$.

(3) $\phi \in \Psi_{k}$ switches controls on the same (transversal) submanifolds as $\phi_{t}(x, \mu)$ and reaches $Q_{f}$. 


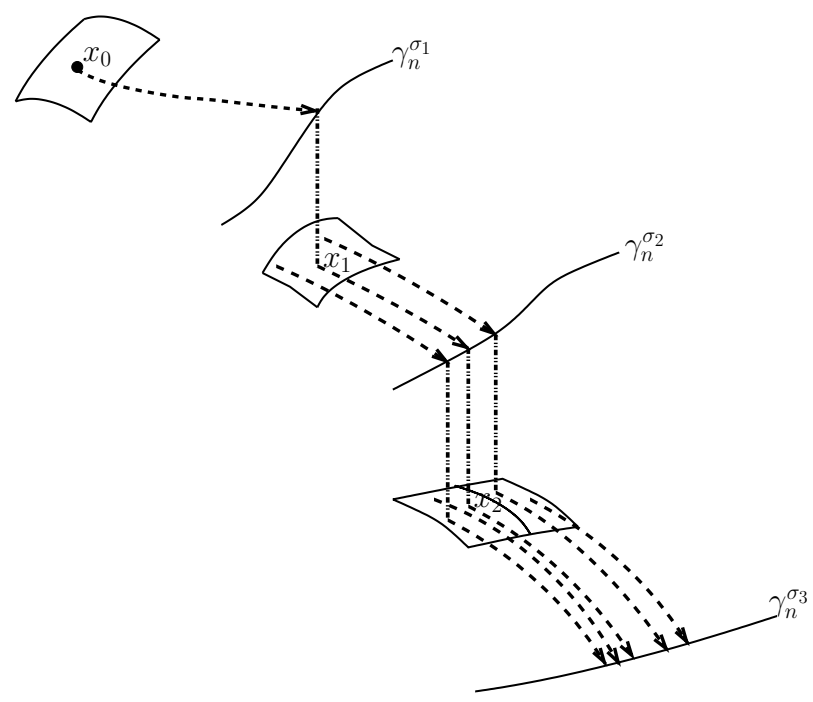

FIG. 6.2. The family of trajectories $\Psi_{k}$ in the proof of Proposition 6.7.

(4) If $x_{j}^{-}=\phi_{\tau_{j}}\left(x_{j-1}, \sigma_{j}\right)$, then $x_{j}$, the initial condition of the next step, satisfies $\left.\left(\left[\left(\sigma_{j}, x_{j}^{-}\right)\right],\left(\sigma_{j+1}, x_{j}\right)\right]\right) \in E$. Thus, the trajectories of $\Psi_{k}$ include jumps at the control switches modeling the nondeterminacy of $A$.

(5) If $\phi \in \Psi_{k}$ intersects $q \in Q$ in $\mathbb{R}^{n}$ at the $j$ th step, then that is the only step where it intersects $q$. Also, all other $\phi^{\prime} \in \Psi_{k}$ that intersect $q$ do so at the $j$ th step only. This requirement can be met for sufficiently large $k$ by the fact that $\phi^{\prime}$ has no self-intersections, by the fact that there are a finite number of steps, and by Remark 6.3(d). For if $\phi^{\prime}$ has a self-intersection, then since $\phi^{\prime}$ approaches $\phi_{t}(x, \mu)$ as $k \rightarrow \infty$, this would imply $\phi_{t}(x, \mu)$ has a self-intersection, contradicting Remark 6.3(c).

The family $\Psi_{k}$ includes all trajectories starting at $x$, using the same sequence of control values as $\mu$, and switching on the same equivalence class boundaries $\phi_{t}(x, \mu)$. Moreover, the initial condition at the start of each step can be any point in an equivalence class that has a nonempty intersection in $\mathbb{R}^{n}$ with the equivalence class reached at the end of the previous step. One visualizes a tube of trajectories that fans out with each successive control switch, as depicted in Figure 6.2. By choosing $k$ sufficiently large and by transversality, all these trajectories reach $Q_{f}$.

Let $W_{k}(\phi)=\sum_{j=1}^{N} L^{2}\left(x_{j-1}, \sigma_{j}\right)+\hat{h}\left(x_{N}\right)$. Observe that for $\phi, \phi^{\prime} \in \Psi_{k}, \mid W_{k}(\phi)-$ $W_{k}\left(\phi^{\prime}\right) \mid \rightarrow 0$ as $k \rightarrow \infty$, using Lipschitz continuity of $L$ and $h$ and Remark 6.3(d). We can define a control policy $\bar{c}$ in which $q \in Q$ is assigned a time step if $q$ is not visited by any trajectory in $\Psi_{k}$. If $q \in Q$ is visited by some $\phi \in \Psi_{k}$ in its $j$ th step, then we assign $\bar{c}(q)=\sigma_{j}$. This gives a well-defined value for $c$ because of Property 4 . By construction, $A$ accepts the time abstract trajectory starting at $q$ corresponding to each trajectory of $\Psi_{k}, \bar{c}$ is admissible because otherwise some time abstract trajectory of $A$ would have a Zeno loop. But a time abstract trajectory of $A$ with a Zeno loop has a corresponding timed trajectory in $\Psi_{k}$ that violates Property 4 of $\Psi_{k}$.

Now we observe that

$$
\hat{J}(q, \bar{c})=\max _{\phi \in \Psi_{k}} W_{k}(\phi):=W_{k}(\bar{\phi}) .
$$

Thus, $\hat{J}_{k}(q, \bar{c})-J_{k}^{2}(x, \mu) \leq\left|W_{k}(\bar{\phi})-W_{k}(\phi(x, \mu))\right| \rightarrow 0$ as $k \rightarrow \infty$. 
Combining Propositions 6.3, 6.6, and 6.7, we have the next theorem.

TheOREM 6.8. For all $x \in \hat{\mathcal{R}}, \hat{V}_{k}(x) \rightarrow V(x)$ as $k \rightarrow \infty$.

7. Implementation. So far we have developed a discrete method for solving an optimal control problem based on hybrid systems and bisimulation. Now we focus on the pragmatic question of how the discretized problem can be efficiently solved. In this section we propose a modification of the Dijkstra algorithm suitable for nondeterministic automata and prove that it is optimal and does not synthesize Zeno loops.

7.1. Motivation. Capuzzo Dolcetta and Evans [12] introduced a method for obtaining approximations of viscosity solutions based on time discretization of the HJB equation. The approximations of the value function correspond to a discrete time optimal control problem, for which an optimal control can be synthesized which is piecewise constant. Finite difference approximations were also introduced in [15] and [47]. In general, the time discretized approximation of the HJB equation is solved by finite element methods. Gonzales and Rofman [23] introduced a discrete approximation by triangulating the domain of the problem, while the admissible control set is approximated by a finite set. Gonzales and Rofman's approach is adapted in several papers, including [18]. The approach of [50] uses the special structure of an optimal control problem to obtain a single-pass algorithm to solve the discrete problem, thus bypassing the expensive iterations of a finite element method. See [45] for a recent adaptation of Tsitsiklis' approach. The essential property needed to find a single pass algorithm is to obtain a partition of the domain so that the cost-to-go value from any equivalence class of the partition is determined from knowledge of the cost-to-go from those equivalence classes with strictly smaller cost-to-go values. We obtain a partition of the domain provided by a bisimulation partition. The combination of the structure of the bisimulation partition and the requirement of non-Zeno trajectories enables us to reproduce the essential property of [50], so that we obtain a Dijkstra-like algorithmic solution. Our approach has complexity $O(N \log N)$ if suitable data structures are used, where $N$ is the number of locations of the finite automaton. The number $N$ is, of course, exponential in $n$, the dimension of the continuous state space.

7.2. Nondeterministic Dijkstra algorithm. The dynamic programming solution (5.4)-(5.5) can be viewed as a shortest path problem on a nondeterministic finite graph subject to all optimal paths satisfying a non-Zeno condition. We propose an algorithm that is a modification of the Dijkstra algorithm for deterministic graphs [17]. First we define the notation. $F_{n}$ is the set of states that have been assigned a control and are deemed finished at iteration $n$, while $U_{n}$ are the unfinished states. At each $n, Q=U_{n} \cup F_{n} . \Sigma_{n}(q) \subseteq \Sigma_{\delta}$ is the set of control events at iteration $n$ that take state $q$ to finished states exclusively. $\tilde{U}_{n}$ is the set of states for which there exists a control event that can take them to finished states exclusively. $\tilde{V}_{n}(q)$ is a tentative cost-to-go value at iteration $n$. $B_{n}$ is the set of best states among $\tilde{U}_{n}$.

The nondeterministic Dijkstra (NDD) algorithm first determines $\tilde{U}_{n}$ by checking if any $q$ in $U_{n}$ can take a step to states belonging exclusively to $F_{n}$. For states belonging to $\tilde{U}_{n}$, an estimate of the value function $\tilde{V}$ following the prescription of (5.4) is obtained: among the set of control events constituting a step into states in $F_{n}$, select the event with the lowest worst-case cost. Next, the algorithm determines $B_{n}$, the states with the lowest $\tilde{V}$ among $\tilde{U}_{n}$, and these are added to $F_{n+1}$. The iteration counter is incremented until it reaches $N=|Q|$. It is assumed in the following description that initially $\hat{V}(q)=\infty$ and $c(q)=\emptyset$ for all $q \in Q$. 


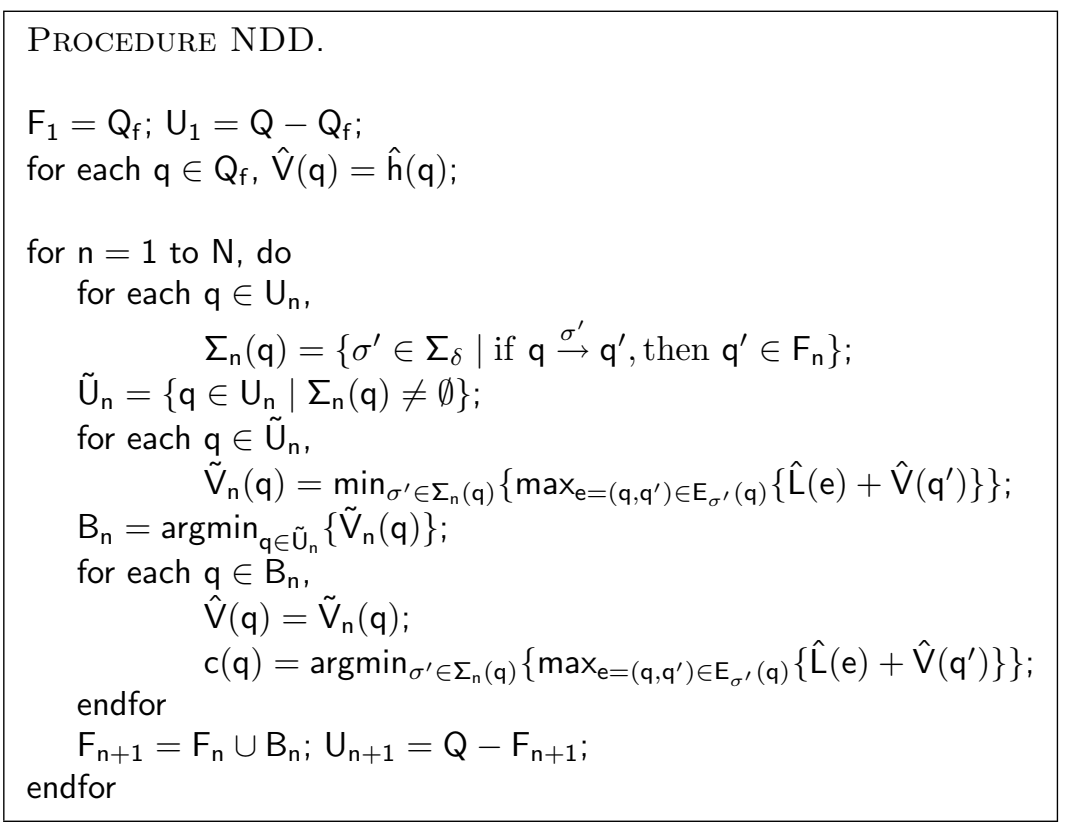

7.3. Justification. In this section we prove that the algorithm is optimal; that is, it synthesizes a control policy so that each $q \in Q$ reaches $Q_{f}$ with the best worstcase cost. We observe a few properties of the algorithm. First, if all states of $Q$ can reach $Q_{f}$ in a nondeterministic sense, then $Q-Q_{f}=\cup_{n} B_{n}$. By nondeterministic sense we mean that for each $q \in Q$, there exists a control policy $c$ such that $\Pi_{c}(q)=\tilde{\Pi}_{c}(q)$. Note that if this condition is not met, then it can happen that at some iteration of NDD, $\tilde{U}_{n}=\emptyset$ but $U_{n} \neq \emptyset$. Second, as in the deterministic case, the algorithm computes $\hat{V}$ in order of level sets of $\hat{V}$. In particular, $\hat{V}\left(B_{n}\right) \leq \hat{V}\left(B_{n+1}\right)$. Finally, we need the following property.

Lemma 7.1. For all $q \in Q$ that can reach $Q_{f}$ in a nondeterministic sense and for all $\sigma^{\prime} \in \Sigma_{\delta}$,

$$
\hat{V}(q) \leq \max _{e=\left(q, q^{\prime}\right) \in E_{\sigma^{\prime}}(q)}\left\{\hat{L}(e)+\hat{V}\left(q^{\prime}\right)\right\} .
$$

Proof. Fix $q \in Q$ and $\sigma^{\prime} \in \Sigma_{\delta}$. There are two cases.

Case 1.

$$
\hat{V}(q) \leq \max _{e=\left(q, q^{\prime}\right) \in E_{\sigma^{\prime}}(q)}\left\{\hat{V}\left(q^{\prime}\right)\right\} .
$$

In this case the result is obvious.

Case 2.

$$
\hat{V}(q)>\max _{e=\left(q, q^{\prime}\right) \in E_{\sigma^{\prime}}(q)}\left\{\hat{V}\left(q^{\prime}\right)\right\} .
$$

By assumption, $q$ belongs to some $B_{n}$. Suppose without loss of generality that $q \in B_{j}$. Together with (7.1) this implies $q^{\prime} \in F_{j}$ for all $q^{\prime}$ such that $q \stackrel{\sigma^{\prime}}{\rightarrow} q^{\prime}$. This, in turn, means that $\sigma^{\prime} \in \Sigma_{j}(q)$ and according to the algorithm

$$
\hat{V}(q)=\tilde{V}_{j}(q) \leq \max _{e=\left(q, q^{\prime}\right) \in E_{\sigma^{\prime}}(q)}\left\{\hat{L}(e)+\hat{V}\left(q^{\prime}\right)\right\},
$$

which proves the result. 
THEOREM 7.2. Algorithm NDD is optimal and synthesizes a control policy with no Zeno loops.

Proof. First we prove optimality. Let $V(q)$ be the optimal (best worst-case) cost-to-go for $q \in Q$ and $\bar{Q}=\{q \in Q \mid V(q)<\hat{V}(q)\}$. Let $l\left(\pi_{q}\right)$ be the number of edges taken by the shortest optimal (best worst-case) trajectory $\pi_{q}$ from $q$. Define $\bar{q}=\arg \min _{q \in \bar{Q}}\left\{l\left(\pi_{q}\right)\right\}$. Suppose that the best worst-case trajectory starting at $\bar{q}$ is $\pi_{\bar{q}}=\bar{q} \stackrel{\sigma^{\prime}}{\rightarrow} \overline{\bar{q}} \rightarrow \cdots$. We showed in the previous lemma that

$$
\hat{V}(\bar{q}) \leq \max _{e=\left(\bar{q}, q^{\prime}\right) \in E_{\sigma^{\prime}}(\bar{q})}\left\{\hat{L}(e)+\hat{V}\left(q^{\prime}\right)\right\}=\hat{L}(e)+\hat{V}(\overline{\bar{q}}) .
$$

Since $\pi_{\bar{q}}$ is the best worst-case trajectory from $\bar{q}$ and by the optimality of $V(\bar{q})$,

$$
V(\bar{q})=\max _{e=\left(\bar{q}, q^{\prime}\right) \in E_{\sigma^{\prime}}(\bar{q})}\left\{\hat{L}(e)+V\left(q^{\prime}\right)\right\}=\hat{L}(e)+V(\overline{\bar{q}}) .
$$

Since $\pi_{\bar{q}}$ is the shortest best worst-case trajectory, we know that $\overline{\bar{q}} \notin \bar{Q}$, so $V(\overline{\bar{q}})=$ $\hat{V}(\overline{\bar{q}})$. This implies $\hat{V}(\bar{q}) \leq \hat{L}(e)+V(\overline{\bar{q}})=V(\bar{q})$, a contradiction.

To prove that the algorithm synthesizes a policy with no Zeno loops, we argue by induction. The claim is obviously true for $F_{1}$. Suppose that the states of $F_{n}$ have been assigned controls forming no Zeno loops. Consider $F_{n+1}$. Each state of $B_{n}$ takes either a time step or a control switch to $F_{n}$, so there cannot be a Zeno loop in $B_{n}$. The only possibility is for some $q \in B_{n}$ to close a Zeno loop with states in $F_{n}$. This implies there exists a control assignment that allows an edge from $F_{n}$ to $q$ to be taken, but this is not allowed by NDD. Thus, $F_{n+1}$ has no Zeno loops.

8. Examples. We consider two simple examples where the solution of the optimal control problem is known in order to illustrate the correctness of the method. The software that generates the optimal enabling conditions is broken into two programs, one that generates the automaton given the information about the bisimulation and one that runs the algorithm NDD. The first program takes as input the control values $\Sigma_{\delta}$ and the level values of $\gamma_{i}^{\sigma}, i=1, \ldots, n, \sigma \in \Sigma_{\delta}$, defining the bisimulation. The functions $\gamma_{i}^{\sigma}, \hat{L}$, and $\hat{h}$ are compiled with the executable. A data structure that associates to each location of the finite automaton the lower and upper level values of each $\gamma_{i}^{\sigma}$ allows time steps to be encoded symbolically, namely, by sorting nodes with equal upper and lower first integral level values in ascending order of $\gamma_{n}^{\sigma}$ level values. The edges of the finite automaton that correspond to $\sigma$-steps are generated numerically by evaluating $\gamma_{i}^{\sigma}$ for $i=1, \ldots, n$ and each $\sigma \in \Sigma_{\delta}$ and thereby determining which equivalence classes overlap for each pair $\left(l, l^{\prime}\right)$ of locations. In our implementation the grid of sample points is $\left\{x \in \Omega, \gamma_{i} \in C^{k}\right\}$ in order to correlate with the mesh size of the bisimulation partition. This numerical step can also be performed symbolically if the functions $\gamma_{i}^{\sigma}$ are polynomials using a quantifier elimination algorithm [5]. However, the quantifier elimination step is expensive, and for approximate solutions it suffices to use a numerical approach.

First we apply our method to Examples 3.1 and 4.1. The bang-bang solution obtained using Pontryagin's maximum principle is well known to involve a single switching curve. The continuous value function $V$ is shown in Figure 8.1(a).

The results of algorithm NDD are shown in Figures 8.1(b) and 8.2. In Figure 8.2 the dashed line is the smooth switching curve for the continuous problem. The black dots identify equivalence classes where NDD assigns a control switch. Considering $g_{e_{-1}}$ we see that the boundary of the enabling condition in the upper left corner is a 


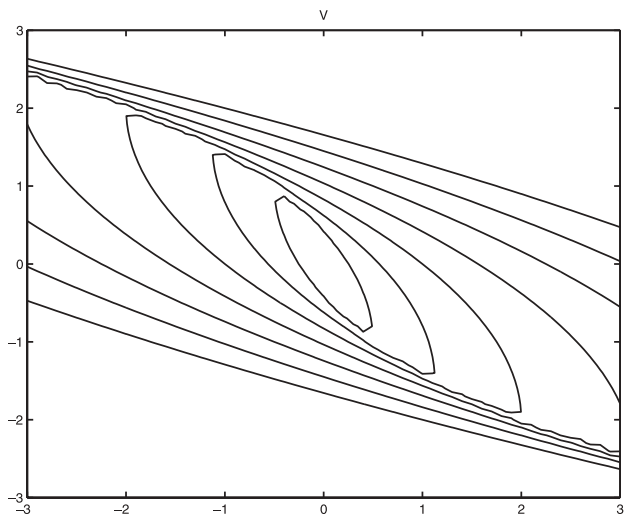

(a) V

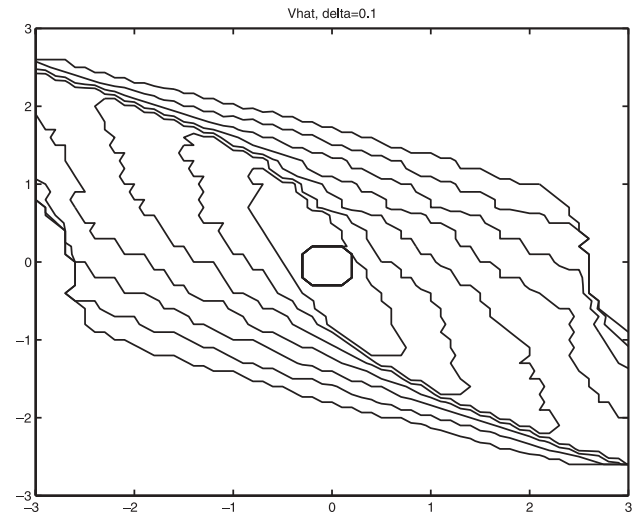

(b) $\hat{V}$ for $\Delta=0.1$.

FIG. 8.1. Continuous and discrete value functions for double integrator.

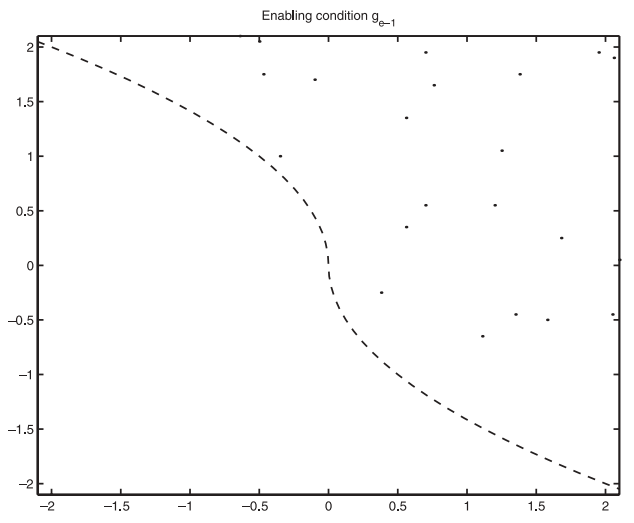

(a) $g_{e_{-1}}$

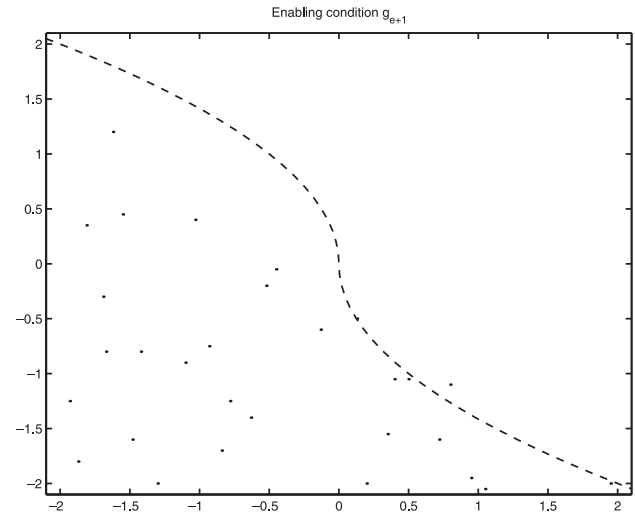

(b) $g_{e_{1}}$

FIG. 8.2. Enabling conditions.

jagged approximation using equivalence classes of the smooth switching curve. Initial conditions in the upper left corner just inside the enabling condition must switch to a control of $u=-1$; otherwise the trajectory will increase in the $x_{2}$ direction and not reach the target. Initial conditions in the upper left corner just outside the enabling condition must allow time to pass until they reach the enabling condition, for if they switched to $u=-1$ they would be unable to reach the target. Hence the upper left boundary of the enabling condition is crisp. The lower right side of the enabling condition which has islands of time steps shows the effect of the nondeterminacy of automaton $A$. These additional time steps occur because it can be less expensive to take a time step than to incur the cost of the worst-case control switch. Indeed consider an initial condition in Figure 8.2(a) which lies in an equivalence class that takes a time step but should take a control switch according to the continuous optimal 


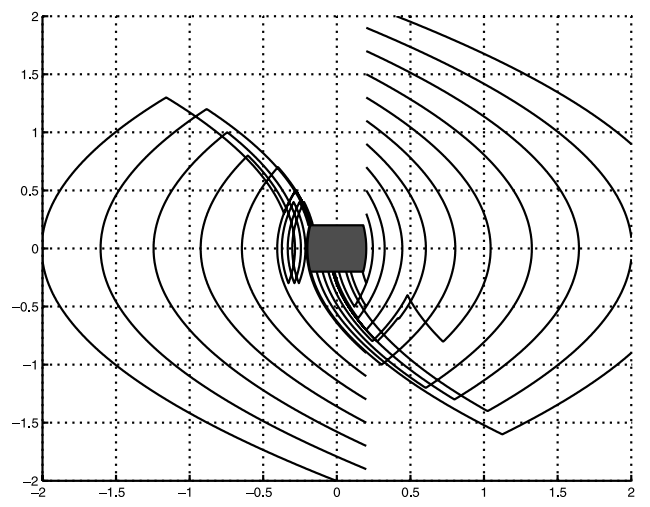

FIG. 8.3. Trajectories of the closed-loop system.

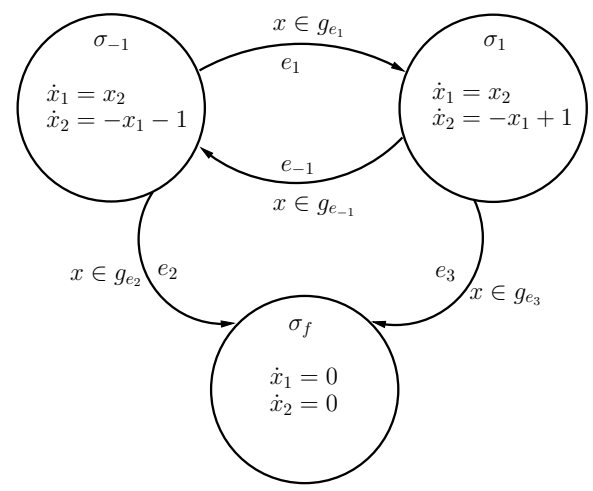

FIG. 8.4. Hybrid automaton for example 2.

control. Such a point will move up and to the left before it takes a control switch. By moving slightly closer to the target, the worst-case cost-to-go incurred in a control switch is reduced. Notice that all such initial conditions eventually take a control switch. This phenomenon of extra time steps is a function of the mesh size $\delta$ : as $\delta$ decreases there are fewer extra time steps. Finally we note that the two enabling conditions have an empty intersection, as expected to ensure non-Zeno trajectories.

Figure 8.3 shows trajectories of the closed-loop system using the controller synthesized by NDD. The central shaded region is an enlarged target set.

Next we consider the time optimal control problem for the system

$$
\begin{aligned}
& \dot{x}_{1}=x_{2}, \\
& \dot{x}_{2}=-x_{1}+u .
\end{aligned}
$$

Suppose $\Omega=(-1,1) \times(-1,1)$ and $\Omega_{f}=\bar{B}_{\epsilon}(0)$, the closed epsilon ball centered at 0 . The cost-to-go function is $J(x, \mu)=\int_{0}^{T(x, \mu)} d t$ and $U=\{u:|u| \leq 1\}$. We select $\Sigma_{\delta}=\{-1,1\}$ so that $\delta=1$. The hybrid system is show in Figure 8.4. The state set is $\left\{\sigma_{-1}=-1, \sigma_{1}=1, \sigma_{f}\right\} \times \mathbb{R}^{2} . g_{e_{-1}}$ and $g_{e_{1}}$ are unknown and must be synthesized, while $g_{e_{2}}=g_{e_{3}}=\Omega_{f}$. A first integral for (8.1) is $\sqrt{\left(x_{1}-u\right)^{2}+x_{2}^{2}}=c_{1}$, where $u= \pm 1$. The transverse foliation is chosen to be defined by the function $\arctan \left(\frac{x_{2}}{x_{1}-u}\right)=c_{2}$. Partitions for locations $\sigma_{1}$ and $\sigma_{-1}$ are shown in Figure 8.5. The results of algorithm NDD are shown in Figures 8.6(b) and 8.7. In Figure 8.7 the dashed line is the 


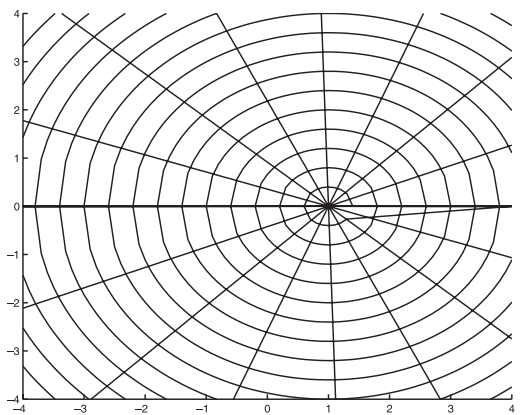

(a) $\sigma_{1}$

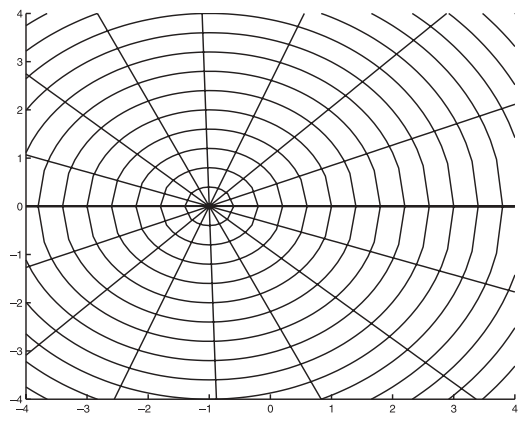

(b) $\sigma_{-1}$

FIG. 8.5. Partitions for states $\sigma_{1}$ and $\sigma_{-1}$ of the hybrid automaton of Figure 8.5.

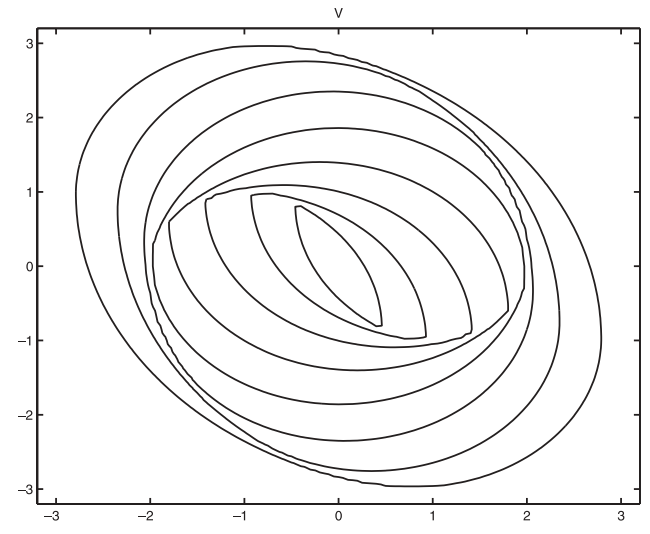

(a) $\mathrm{V}$

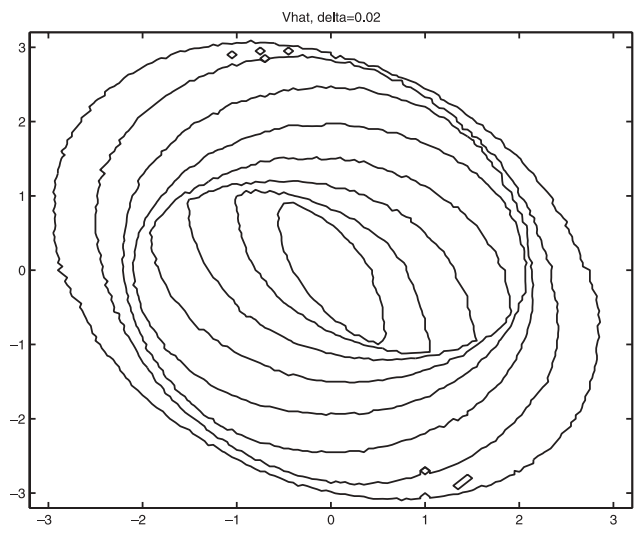

(b) $\hat{V}$ for $\Delta=0.02$.

FIG. 8.6. Continuous and discrete value functions for example 2.

switching curve for the continuous problem. As in the previous example the black dots identify equivalence classes where NDD assigns a control switch. Figure 8.8 shows trajectories of the closed-loop system using the controller synthesized by NDD. An enlarged target set is at the origin.

Remark 8.1. From these examples we observe that our method is best suited to problems when there are relatively few control switches, as each control switch incurs an error of order $\delta$. Also the method is suited to problems where bang-bang controls are used. The method has advantages in situations where a fine time discretization of the vector field is needed for standard finite element methods. We do not require time discretization because of the particular choice of grid, which captures time evolution exactly. Finally, because the method requires computation of weak first integrals, only systems for which first integrals are computable in closed form are considered.

Table 8.1 shows the computation times for the two examples as a function of $\delta$. The automaton size and the time in seconds to generate it appear in the second and 


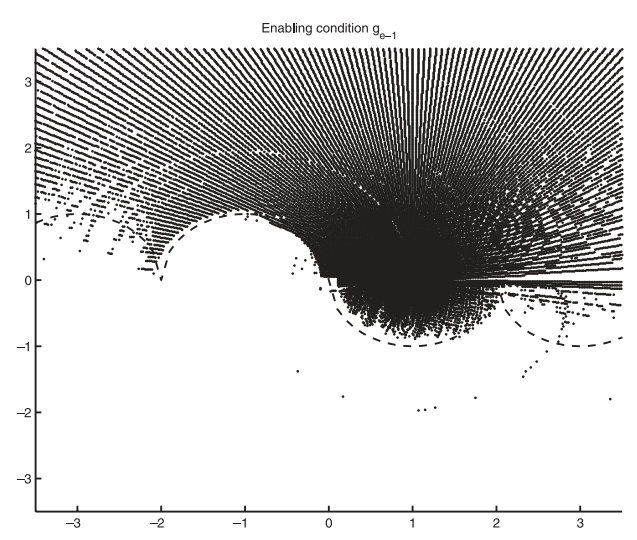

(a) $g_{e_{-1}}$

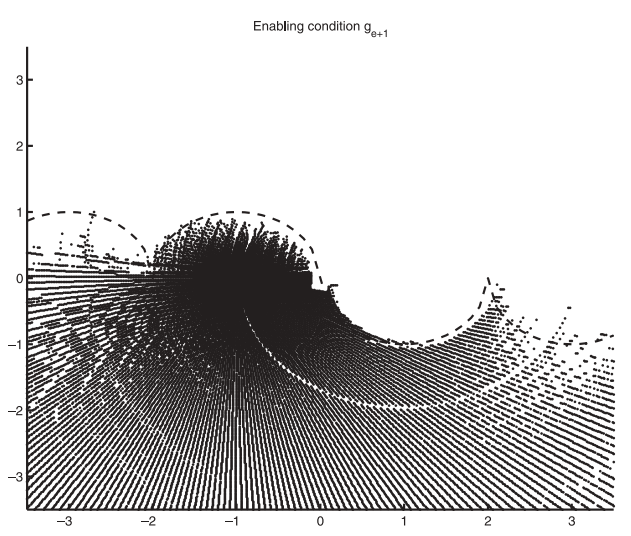

(b) $g_{e_{1}}$

FIG. 8.7. Enabling conditions for example 2.

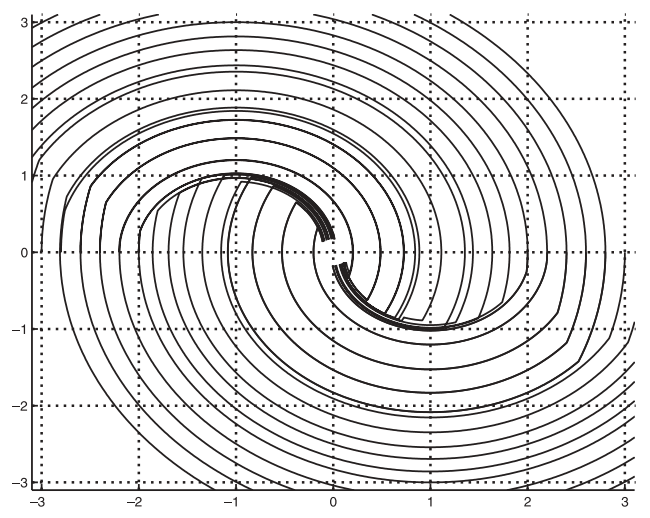

FIG. 8.8. Trajectories of the closed-loop system for example 2.

TABLE 8.1

\begin{tabular}{|l|l|l|l|l|l|l|}
\hline Example & \multicolumn{1}{|c|}{$\delta$} & \multicolumn{1}{|c|}{$N$} & Automaton & I/O & NDD & Finished \\
\hline 1 & .2 & 7200 & .11 & 4.57 & .02 & 3274 \\
\hline 1 & .1 & 28800 & .46 & 5.69 & .08 & 12835 \\
\hline 1 & .05 & 115200 & 1.97 & 9.09 & .38 & 51490 \\
\hline 1 & .025 & 460800 & 7.83 & 22.91 & 1.77 & 205624 \\
\hline 2 & .2 & 1920 & .03 & 4.42 & .01 & 1920 \\
\hline 2 & .1 & 7560 & .09 & 4.78 & .07 & 7560 \\
\hline 2 & .05 & 30240 & .39 & 6.13 & .28 & 30240 \\
\hline 2 & .025 & 120960 & 1.55 & 11.04 & 1.3 & 189000 \\
\hline 2 & .0125 & 482880 & 7.99 & 35.29 & 7.12 & 482880 \\
\hline
\end{tabular}


third columns. We report the time for file I/O, which otherwise would dominate the computation times. The time in seconds to run NDD and the size of the set $F_{n}$ of finished states appear in the last two columns. Note that not all nodes are finished in the first example because the regions of $\mathbb{R}^{2}$ that are partitioned in the two locations do not overlap perfectly, resulting in the nonexistence of a trajectory that can reach the origin starting in a subset of the nonoverlapping areas.

9. Conclusion. In this paper we have developed a methodology for the synthesis of optimal controls based on hybrid systems and bisimulations. The idea is to translate the optimal control problem to a switching problem on a hybrid system whose locations describe the dynamics when the control is constant. When the vector fields for each location of the hybrid automaton have local first integrals which can be expressed analytically we are able to define a finite bisimulation using the approach of [10]. From the finite bisimulation we obtain a (time abstract) finite automaton on which a dynamic programming problem can be formulated that can be solved efficiently. We proposed an efficient single-pass algorithm to solve this dynamic programming problem and demonstrated its correctness on two simple examples.

\section{REFERENCES}

[1] R. Alur And D. Dill, A theory of timed automata, Theoret. Comput. Sci., 126 (1994), pp. 183235.

[2] R. Alur, T. Henzinger, G. Lafferriere, and G. Pappas, Discrete abstractions of hybrid systems, Proc. IEEE, 88 (2000), pp. 971-984.

[3] V.I. ARnold, Mathematical Methods in Classical Mechanics, Springer-Verlag, New York, 1997.

[4] M. Bardi and I. Capuzzo-Dolcetta, Optimal Control and Viscosity Solutions of HamiltonJacobi-Bellman Equations, Birkhäuser, Boston, 1997.

[5] S. Basu, R. Pollack, AND M.-F. Roy, On the combinatorial and algebraic complexity of quantifier elimination, J. ACM, 43 (1996), pp. 1002-1045.

[6] A. Bensoussan and J.L. Menaldi, Hybrid control and dynamic programming, Dynam. Contin. Discrete Impuls. Systems, 3 (1997), pp. 395-442.

[7] G. Bluman And S. Anco, Symmetry and Integration Methods for Differential Equations, Springer-Verlag, New York, 2002.

[8] V.G. BoltyanskiI, Sufficient conditions for optimality and the justification of the dynamic programming method, SIAM J. Control, 4 (1966), pp. 326-361.

[9] M. Branicky, V. Borkar, and S. Mitter, A unified framework for hybrid control: Model and optimal control theory, IEEE Trans. Automat. Control, 43 (1998), pp. 31-45.

[10] M. BRoucKe, A geometric approach to bisimulation and verification of hybrid systems, in Hybrid Systems: Computation and Control, F. Vaandrager and J. van Schuppen, eds., Lecture Notes in Comput. Sci. 1569, Springer-Verlag, New York, 1999, pp. 61-75.

[11] M. Broucke, Reachability analysis for hybrid systems with linear dynamics, in Proceedings of the Fifteenth International Symposium, Mathematical Theory of Networks and Systems (MTNS '02), Notre Dame, IN, 2002.

[12] I. Capuzzo Dolcetta And L.C. Evans, Optimal switching for ordinary differential equations, SIAM J. Control Optim., 22 (1984), pp. 143-161.

[13] J. Chavarriga, H. Giacomini, J. Gine, and J. Llibre, On the integrability of two-dimensional flows, J. Differential Equations, 157 (1999), pp. 163-182.

[14] M. Crandall and P. Lions, Viscosity solutions of Hamilton-Jacobi equations, Trans. Amer. Math. Soc., 277 (1983), pp. 1-42.

[15] M.G. Crandall and P.L. Lions, Two approximations of solutions of Hamilton-Jacobi equations, Math. Comp., 43 (1984), pp. 1-19.

[16] P.E. Crouch and R.L. Grossman, The explicit computation of integration algorithms and first integrals for ordinary differential equations with polynomial coefficients using trees, in Proceedings of International System Symposium on Symbolic and Algebraic Computation (ISSAC '92), P.S. Wang, ed., ACM, New York, 1992, pp. 89-94.

[17] E.W. Dijkstra, A note on two problems in connection with graphs, Numer. Math., 1 (1959), pp. 269-271. 
[18] M. FALCONE, A numerical approach to the infinite horizon problem of deterministic control theory, Appl. Math. Optim., 15 (1987), pp. 1-13.

[19] K. Feng And D.-L. Wang, Dynamical Systems and Geometric Construction of Algorithms, in Computational Mathematics in China, Contemp. Math. 163, Z. Shi and C. Yang, eds., AMS, Providence, RI, 1994.

[20] W.H. Fleming And R.W. Rishel, Deterministic and Stochastic Optimal Control, SpringerVerlag, New York, 1975.

[21] A.T. Fuller, Study of an optimum non-linear control system, J. Electron. Control, 15 (1963), pp. 63-71.

[22] J. Goldman, Integrals of multinomial systems of ordinary differential equations, J. Pure Appl. Algebra, 45 (1987), pp. 225-240.

[23] R. Gonzalez and E. Rofman, On deterministic control problems: An approximation procedure for the optimal cost I: The stationary problem, SIAM J. Control Optim., 23 (1985), pp. 242266.

[24] A. Goriely, Integrability and Nonintegrability of Dynamical Systems, World Scientific, River Edge, NJ, 2001.

[25] E. HAIRER, Geometric integration of ordinary differential equations on manifolds, BIT, 41 (2001), pp. 996-1007.

[26] T. Henzinger, Hybrid automata with finite bisimulations, in Proceedings of the 22nd ICALP: Automata, Languages and Programming, Lecture Notes in Comput. Sci. 944, SpringerVerlag, New York, 1995, pp. 324-335.

[27] W. Hereman, Symbolic software for Lie symmetry analysis, in CRC Handbook of Lie Group Analysis of Differential Equations, N.H. Ibragimov, ed., CRC Press, Boca Raton, FL, 1995.

[28] A. Iserles And A. Zanna, Preserving algebraic invariants with Runge-Kutta methods, J. Comput. Appl. Math., 125 (2000), pp. 69-81.

[29] I. KupKa, The ubiquity of the Fuller phenomenon, in Nonlinear Controllability and Optimal Control, H.J. Sussmann, ed., Marcel Dekker, New York, 1990.

[30] H.B. Lawson, The Qualitative Theory of Foliations, in Regional Conf. Ser. Math. 27, AMS, Providence, RI, 1977.

[31] W. LeVeque, Fundamentals of Number Theory, Addison-Wesley, Reading, MA, 1977.

[32] P.L. Lions, Generalized Solutions of Hamilton-Jacobi Equations, Pitman, Boston, 1982.

[33] O. Maler, A. Pnueli, and J. Sifakis, On the synthesis of discrete controllers for timed systems, in Proceedings of STACS '95, E.W. Mayr and C. Puech, eds., Lecture Notes in Comput. Sci. 900, Springer-Verlag, New York, 1995, pp. 229-242.

[34] Y.-K. MAN, Computing closed form solutions of first order ODE's using the Prelle-Singer procedure, J. Symbolic Comput., 16 (1993), p. 423.

[35] Y.K. MAN, First integrals of autonomous systems of differential equations and the Prelle-Singer procedure, J. Phys. A, 27 (1994), pp. L329-L332.

[36] R. MCLachlan And C. Scovel, A survey of open problems in symplectic integration, in Integration Algorithms and Classical Mechanics, J.E. Marsden, G. Patrick, and W. Shadwick, eds., AMS, Providence, RI, 1996, pp. 151-180.

[37] P. Olver, Application of Lie Groups to Differential Equations, Springer-Verlag, New York, 1986.

[38] L.S. Pontryagin, V. Boltiansky, A. Gamkrelitze, and E. Mischenko, The Mathematical Theory of Optimal Processes, Interscience Publishers, New York, 1962.

[39] M.J. Prelle and M.F. Singer, Elementary first integrals of differential equations, Trans. Amer. Math. Soc., 279 (1983), pp. 215-229.

[40] G. Quispel AND H. CAPEL, Solving ODE's numerically while preserving a first integral, Phys. Lett. A, 218 (1996), pp. 223-228.

[41] J. RAISCH, Controllability and observability of simple hybrid control systems-FDLTI plants with symbolic measurements and quantized control inputs, in International Conference on Control '94, vol. 1, IEE, London, 1994, pp. 595-600.

[42] H. Royden, Real Analysis, 3rd ed., Prentice-Hall, Englewood Cliffs, NJ, 1988.

[43] F. SCHWARZ, A REDUCE package for determining first integrals of autonomous systems of ordinary differential equations, Comput. Phys. Comm., 39 (1986), pp. 285-296.

[44] F. SCHWARZ, An algorithm for determining polynomial first integrals of autonomous systems of ordinary differential equations, J. Symbolic Comput., 1 (1985), pp. 229-233.

[45] J.A. Sethian and A. Vladimirsky, Ordered upwind methods for static Hamilton-Jacobi equations: Theory and algorithms, SIAM J. Numer. Anal., 41 (2003), pp. 325-363.

[46] W.Y. Sit, On Goldman's algorithm for solving first-order multinomial autonomous systems, in Proceedings of Applied Algebra, Algebraic Algorithms and Error-Correcting Codes, 6th International Conference, T. Mora, ed., Springer-Verlag, New York, 1989, pp. 386-395. 
[47] P.E. Souganidis, Approximation schemes for viscosity solutions of Hamilton-Jacobi equations, J. Differential Equations, 59 (1985), pp. 1-43.

[48] J. Stiver, P. Antsaklis, And M. Lemmon, A logical DES approach to the design of hybrid control systems, Math. Comput. Modelling, 23 (1996), pp. 55-76.

[49] J.-M. Strelcyn and S. Wojciechowski, A method of finding first integrals for threedimensional dynamical systems, Phys. Lett. A, 133 (1988), pp. 207-212.

[50] J.N. TsitsikLis, Efficient algorithms for globally optimal trajectories, IEEE Trans. Automat. Control, 40 (1995), pp. 1528-1538.

[51] H.S. WitsenhaUSEn, A class of hybrid-state continuous-time dynamic systems, IEEE Trans. Automat. Control, 11 (1966), pp. 161-167.

[52] H. WonG-ToI, The synthesis of controllers for linear hybrid automata, in Proceedings of the 36th IEEE Conference on Decision and Control, San Diego, CA, 1997, pp. 4607-4612.

[53] X. YAng, M. Lemmon, And P. Antsaklis, On the supremal controllable sublanguage in the discrete-event model of nondeterministic hybrid control systems, IEEE Trans. Automat. Control, 40 (1995), pp. 2098-2103. 